\title{
The ionized gas in the central region of NGC 5253
}

\section{D mapping of the physical and chemical properties $\star$}

\author{
A. Monreal-Ibero ${ }^{1}$, J. R. Walsh ${ }^{2}$, and J. M. Vílchez ${ }^{1}$ \\ 1 Instituto de Astrofísica de Andalucía (CSIC), C/ Camino Bajo de Huétor, 50, 18008 Granada, Spain \\ e-mail: ami@iaa.es \\ 2 European Southern Observatory, Karl-Schwarzschild Strasse 2, 85748 Garching bei München, Germany
}

Received 4 May 2012 / Accepted 8 June 2012

\begin{abstract}
Context. Blue compact dwarf (BCD) galaxies constitute the ideal laboratories to test the interplay between massive star formation and the surrounding gas. As one of the nearest BCD galaxies, NGC 5253 was previously studied with the aim to elucidate in detail the starburst interaction processes. Some open issues regarding the properties of its ionized gas still remain to be addressed.

Aims. The 2D structure of the main physical and chemical properties of the ionized gas in the core of NGC 5253 has been studied. Methods. Optical integral field spectroscopy (IFS) data has been obtained with FLAMES Argus and lower resolution gratings of the Giraffe spectrograph.

Results. We derived 2D maps for different tracers of electron density $\left(n_{\mathrm{e}}\right)$, electron temperature $\left(T_{\mathrm{e}}\right)$ and ionization degree. The maps for $n_{\mathrm{e}}$ as traced by [O II], [S II], [Fe III], and [Ar IV] line ratios are compatible with a 3D stratified view of the nebula with the highest $n_{\mathrm{e}}$ in the innermost layers and a decrease of $n_{\mathrm{e}}$ outwards. 2D maps of $T_{\mathrm{e}}$ were measured from [O III] and [S II] line ratios; to our knowledge, this is the first time that a $T_{\mathrm{e}}$ map based on [S II] lines for an extragalactic object has been presented. The joint interpretation of the $T_{\mathrm{e}}\left(\left[\mathrm{S}_{\mathrm{II}}\right]\right)$ and $T_{\mathrm{e}}([\mathrm{O} \mathrm{III}])$ maps is consistent with a $T_{\mathrm{e}}$ structure in $3 \mathrm{D}$ with higher temperatures close to the main ionizing source surrounded by a colder and more diffuse component. The highest ionization degree is found at the peak of emission for the gas with relatively high ionization in the main Giant $\mathrm{H}_{\text {II }}$ Region and lower ionization degree delineating the more extended diffuse component. We derived abundances of oxygen, neon, argon, and nitrogen. Abundances for $\mathrm{O}, \mathrm{Ne}$ and Ar are constant over the mapped area within $\lesssim 0.1$ dex. The mean $12+\log (\mathrm{O} / \mathrm{H})$ is $8.26 \pm 0.04$ while the relative abundances of $\log (\mathrm{N} / \mathrm{O})$, $\log (\mathrm{Ne} / \mathrm{O})$ and $\log (\mathrm{Ar} / \mathrm{O})$ were $\sim-1.32 \pm 0.05,-0.65 \pm 0.03$ and $-2.33 \pm 0.06$, respectively. There are two locations with enhanced $\mathrm{N} / \mathrm{O}$. The first $(\log (\mathrm{N} / \mathrm{O}) \sim-0.95)$ occupies an area of about $80 \mathrm{pc} \times 35 \mathrm{pc}$ and is associated to two super star clusters. The second $(\log (\mathrm{N} / \mathrm{O}) \sim-1.17)$, reported here for the first time, is associated to two moderately massive $\left(2-4 \times 10^{4} M_{\odot}\right)$ and relatively old ( $\sim 10 \mathrm{Myr}$ ) clusters. A comparison of the N/O map with those produced by strong line methods supports the use of N2O2 over N2S2 in the search for chemical inhomogeneities within a galaxy. The results on the localized nitrogen enhancement were used to compile and discuss the factors that affect the complex relationship between Wolf-Rayet stars and N/O excess.
\end{abstract}

Key words. galaxies: starburst - galaxies: dwarf - galaxies: individual: NGC 5253 - galaxies: ISM - galaxies: abundances galaxies: kinematics and dynamics

\section{Introduction}

Local blue compact dwarf galaxies (BCDs, see Kunth \& Östlin 2000 , for a review) constitute ideal laboratories to test how the interaction between massive star formation, gas and dust affects galaxy evolution. Specifically, both winds of massive stars and supernova explosions i) inject mechanical energy to the interstellar medium (ISM), redistributing the gas within the galaxy and therefore, quenching (or igniting) future star formation; ii) eject processed material into the ISM thus causing a chemical enrichment of the galaxy.

NGC 5253 is an example BCD particularly suited for the study of the interaction between gas and massive star formation, since it is relatively close and a wealth of ancillary information is available in all spectral ranges, from X-ray to radio. This galaxy belongs to the Centaurus A/M 83 complex (Karachentsev et al. 2007) and is suffering a recent burst of star formation. The existence of an $\mathrm{H}_{\mathrm{I}}$ plume extending along the optical minor axis

* Based on observations collected at the European Organisation for Astronomical Research in the Southern Hemisphere, Chile (ESO Programme 078.B-0043 and 383.B-0043).
(Kobulnicky \& Skillman 2008) supports the idea of the burst being caused by a former encounter with M 83 (van den Bergh 1980). A wealth of studies at different wavelengths shows that this galaxy is peculiar in several aspects. In particular, high resolution multiband photometry with the Hubble Space Telescope (HST) revealed the existence of several relatively young star clusters in the central region of the galaxy with typical masses of $\sim 2-120 \times 10^{3} M_{\odot}$ (e.g. Harris et al. 2004). Among those, two very massive $\left(\sim 1-2 \times 10^{6} M_{\odot}\right)$ Super Star Clusters (SSCs) at the nucleus of the galaxy, separated by $\sim 0$.' $^{\prime}$ (Alonso-Herrero et al. 2004) and associated with a very dense compact $\mathrm{H}$ in region, only detected in the radio at $1.3 \mathrm{~cm}$ and $2 \mathrm{~cm}$ (Turner et al. 2000), stand out as candidates to be the youngest globular cluster(s) yet observed (Gorjian et al. 2001). Also, López-Sánchez et al. (2012) showed how this galaxy does not satisfy the SchmidtKennicutt law of star formation and seems to be slightly metaldeficient in comparison with starbursts of similar baryonic mass. But probably, the most well-known peculiarity of NGC 5253 is the existence of areas with enhanced abundance of nitrogen. This was first reported by Welch (1970) and confirmed afterwards by several works (e.g. Walsh \& Roy 1989). 


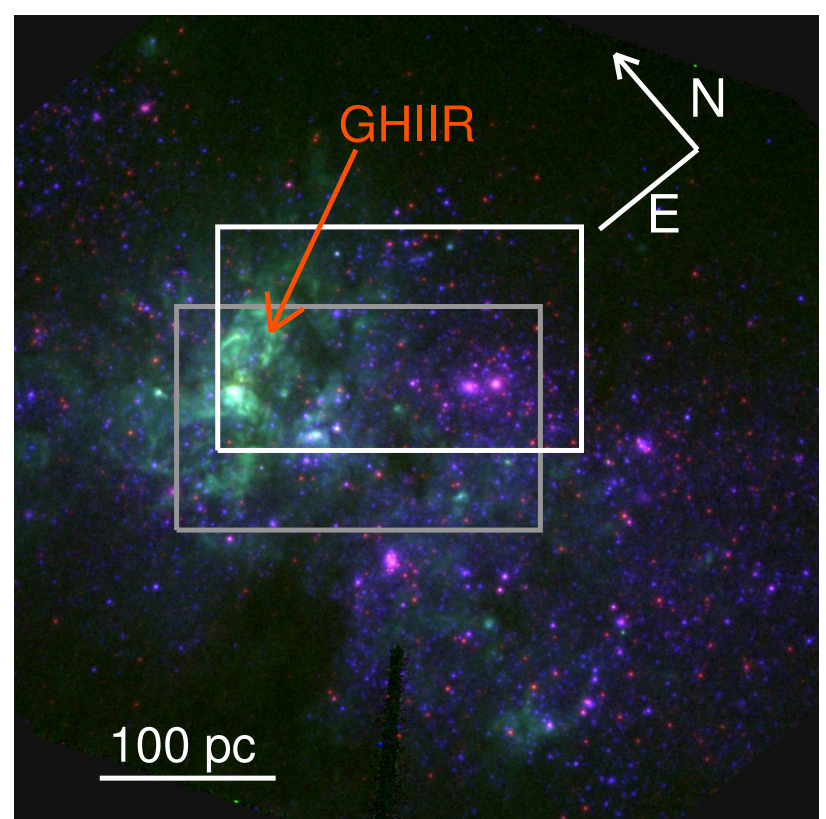

Fig. 1. False colour image in filters $F 435 W$ ( $B$, blue channel), $F 658 N$ (H $\alpha$, green channel), and $F 814 W$ ( $I$, red channel) for NGC 5253 using HST-ACS images (programme 10608, P.I.: Vacca). The area covered in ESO program 383.B-0043 (078.B-0043) is marked with a white (grey) rectangle while the main $\mathrm{GH}$ in of the galaxy is labeled in orange. The orientation and scale for a distance of $3.8 \mathrm{Mpc}$ are indicated.

Narrow band imaging showed that as a consequence of its starburst nature, the ionized gas in NGC 5253 presents a complex structure that includes filaments and arcs (e.g. Calzetti et al. 2004). Therefore, proper characterization of the properties of the ionized gas in NGC 5253 would benefit from high quality twodimensional spectral mapping over a contiguous area of interest. Nowadays, integral field spectroscopy (IFS) facilities represent the obvious choice to obtain this kind of information and several works devoted to the study of BCDs using this approach have been published in recent years (e.g. Izotov et al. 2006a; García-Lorenzo et al. 2008; James et al. 2009; Westmoquette et al. 2010).

In particular, Monreal-Ibero et al. (2010b, hereafter Paper I) carried out a detailed study of the central region in NGC 5253 using IFS data collected with FLAMES (Pasquini et al. 2002). One of the topics studied in more detail there was the kinematics of the ionized gas. We found that the line profiles were complex, needing up to three components to reproduce them. In the main Giant Hir Region (GH IIR, see Fig. 1), one of them presented supersonic widths while the other two were relatively narrow. Moreover, the broad component presented an excess in nitrogen of $\sim 1.4$ times than that of the narrow one (Monreal-Ibero et al. 2012). This was consistent with a scenario where the two SSCs produce an outflow that encounters the previously quiescent gas. Also, the area polluted with extra nitrogen was delimited very precisely, based on the excess of the $\left[\mathrm{N}_{\text {II }}\right] \lambda 6584 / \mathrm{H} \alpha$ ratio with respect to $\left[\mathrm{S}_{\text {II }}\right] \lambda \lambda 6717,6731 / \mathrm{H} \alpha$ and clearly demonstrated that at least part of the Wolf-Rayet (WR) star population cannot be the cause of this enrichment. Moreover, we could resolve a long-standing issue regarding the elusive He II emission at $\lambda 4686 \AA$ A. Campbell et al. (1986) mentioned a possible detection of He II but this result was not certainly confirmed afterwards. We detected several localized areas with clear He II $\lambda 4686$ detection although, rather puzzlingly, not coincident in general with the area exhibiting extra nitrogen.
Table 1. Observation log.

\begin{tabular}{ccccc}
\hline \hline Grating & $\begin{array}{c}\text { Spectral range } \\
(\AA)\end{array}$ & Resolution & $\begin{array}{c}t_{\exp } \\
(\mathrm{s})\end{array}$ & Airmass \\
\hline L385.7 & $3610-4081$ & 12800 & $21 \times 895$ & $1.00-1.10$ \\
L427.2 & $3964-4567$ & 10200 & $9 \times 895$ & $1.00-1.09$ \\
\hline
\end{tabular}

With the limited spectral range utilized in that work, we could only determine relative nitrogen abundances. The most natural explanation for the interpretation of the increase of $\left[\mathrm{N}_{\text {II }}\right] \lambda 6584 /\left[\mathrm{S}_{\text {II }}\right] \lambda \lambda 6717,6731$ was an enhancement in nitrogen abundance; an interpretation supported by previous observational evidence (e.g. Kobulnicky et al. 1997). However, this is by no means the only alternative. Instead, specific combinations of ionization structure and metallicity gradient could also produce similar observables. Since dwarf galaxies are not expected to have strong metallicity gradients in a similar manner to large spirals, this option seems unlikely. However, it could not be rejected a priori in our previous work.

Here, we present the natural continuation of the work in Paper I. To overcome the aforementioned drawbacks, we obtained new FLAMES observations that allowed missing physical properties, such as electron temperature $\left(T_{\mathrm{e}}\right)$ and local degree of excitation, to be mapped. In this manner we are able to: i) map the chemical content in the central part of the galaxy and detect inhomogeneities (if any) in an unbiased manner; ii) evaluate how well relative abundance tracers based on strong emission lines reproduce the values derived from direct measurements; iii) check if the derived physical and chemical structure is consistent with the picture sketched in Paper I.

The paper is organized as follows: Sect. 2 describes the observations and technical details about the data reduction and processing necessary to extract the required information for the analysis. Section 3 contains the results from one Gaussian fitting for the physical (i.e. $T_{\mathrm{e}}, n_{\mathrm{e}}$, ionization degree) and chemical (i.e. metallicity and relative abundances) properties both in 2D and in particularly interesting apertures. Section 4 will explore how the derived maps trace the 3D physical structure of the central part of NGC 5253, and the reliability (or limitations) of strong line based tracers to determine the relative abundance in nitrogen. Basic information for NGC 5253 can be found in Table 1 in Paper I.

\section{Observations and data processing}

\subsection{Observations}

Data were obtained with the Fibre Large Array Multi Element Spectrograph, FLAMES (Pasquini et al. 2002) at Kueyen, Telescope Unit 2 of the $8 \mathrm{~m}$ VLT at ESO's observatory on Paranal. We used the ARGUS Integral Field Unit with the sampling of $0.52^{\prime \prime} /$ lens. This permits coverage of a field of view (fov) of $11^{\prime \prime} .5 \times 7^{\prime \prime}$. 3. Utilized gratings were L385.7 (LR1), L427.2 (LR2), L479.7 (LR3) and L682.2 (LR6).

Data for the two last gratings were obtained in visitor mode on February 10, 2007 (programme 078.B-0043). Details of these observations appear in Paper I. In addition to these, data for the L385.7 (LR1) and L427.2 (LR2) were obtained in service mode during June 2009 (programme 383.B-0043). The spectral range, resolving power, exposure time and airmass for each configuration are listed in Table 1 . Seeing ranged typically between 0.4 and $11^{\prime \prime} 2$ with a median ( \pm standard deviation) of $\sim 00^{\prime} 8( \pm 0.2)$. All the data were taken under clear conditions. In addition, standard 
sets of calibration files were obtained. These included continuum and ThAr arc lamps exposures as well as frames for the spectrophotometric standard stars HR 7596 and LTT 3218 for the L385.7 (LR1) and L427.2 (LR2) gratings respectively.

Due to a guiding problem, there was a $\sim 2$ '. 9 offset between the pointings on runs 078.B-0043 and 383.B-0043. In spite of this offset, the main emitting regions were covered by all the configurations. The precise area covered in each run is shown in Fig. 1 which contains the FLAMES fov over-plotted on an HST $B, \mathrm{H} \alpha, I$ colour image.

\subsection{Data reduction}

The processing of the data on NGC 5253 for run 078.B-0043 is described in Paper I. Those for run 383.B-0043 were processed using a combination of the pipeline provided by ESO (version 2.8.9) ${ }^{1}$ via esorex, version 3.9.0 and some IRAF $^{2}$ routines.

The corresponding master calibration files were created with the ESO pipeline tasks gimasterbias, gimasterflat and giwavecalibration. In particular, fifteen bias frames were used for the masterbias creation while six and three continuum lamp exposures were needed for the creation of the L385.7 and L427.2 masterflats, respectively. In order to remove the cosmic rays in the target exposures, we combined all the individual frames for a given grating using imcombine with IRAF. After that, each combined frame was processed using the ESO pipeline in order to perform bias subtraction, spectral tracing and extraction, wavelength calibration and correction of fibre transmission and data cube creation.

In both settings, four isolated and evenly distributed arc lines were fitted by a Gaussian in each spectrum in order to estimate the uncertainties in the wavelength calibration as well as the instrumental width. Centroids of the lines were determined with an accuracy of $\sim 0.005 \AA$ which, for the covered spectral range, translates into velocities of $\sim 0.3-0.4 \mathrm{~km} \mathrm{~s}^{-1}$. We measured a spectral resolution of $F W H M \sim 0.329 \AA$ and $0.446 \AA$ for the L385.7 and L427.2, respectively. This translates to $\sigma_{\text {instru }} \sim 10.9$ and $13.3 \mathrm{~km} \mathrm{~s}^{-1}$.

In the next step, the sky background was subtracted. For that purpose, we created a good signal-to-noise $(\mathrm{S} / \mathrm{N})$ spectrum by averaging the spectra of the sky fibres in each combined frame which was subtracted from every spectrum. Fibres suffering significant contamination due to cross-talk from adjacent object fibres were excluded from this combination.

Relative flux calibration was performed within IRAF. The sky in the standard star frames was subtracted following the same methodology as with the science frames and then a spectrum was formed for each calibration star by co-adding all the fibres of each standard star frame. Then, a sensitivity function was determined with the IRAF tasks standard and sensfunc and science frames were calibrated with calibrate.

Finally, since data were taken in clear conditions, it was necessary to place both datacubes on the same (relative) flux scale. We scaled each spectrum in the LR1 mode to the same level as that of LR2 using the $\mathrm{H} 7$ and [Ne III] $\lambda 3967$ emission lines, which were present in both instrumental set-ups.

\footnotetext{
1 http://www . eso.org/projects/dfs/dfs-shared/web/vlt/ vlt-instrument-pipelines.html

2 The Image Reduction and Analysis Facility IRAF is distributed by the National Optical Astronomy Observatories which is operated by the association of Universities for Research in Astronomy, Inc. under cooperative agreement with the National Science Foundation.
}

\subsection{Subtracting the emission of the stellar population}

The spectral region analyzed in this work is rich in spectral features caused by the underlying stellar population (e.g. Balmer absorption lines). Their effect on the estimation of the flux in the emission lines of the gas is negligible for the brightest lines and/or those in the area of the bright GH IIR. However, they may affect the measurements in the area of lower surface brightness and, especially in those spaxels associated with the relatively older clusters $(\sim 10 \mathrm{Myr})^{3}$ associated to the peak of emission in the continuum $\sharp 3$ (see Paper I, for the definition of the peaks of emission).

To estimate and correct this effect, we modelled and subtracted out the contribution of the underlying stellar continuum using the STARLIGHT ${ }^{4}$ spectral synthesis code (Cid Fernandes et al. 2005, 2009). This code reproduces a given observed spectrum by selecting a linear combination of a sub-set of $N_{\star}$ spectral components from a pre-defined set of base spectra. In our particular case, we utilized as base spectra a set of single star populations from Bruzual \& Charlot (2003). These are based on the Padova 2000 evolutionary tracks (Girardi et al. 2000) and assume Salpeter initial mass function between 0.1 and $100 M_{\odot}$. Since NGC 5253 has a metallicity of $Z \sim 0.3 Z_{\odot}{ }^{5}$ (e.g. Kobulnicky et al. 1999), we utilized only base spectra with $Z=0.004$ and 0.008 . For each metallicity, we selected a set of 18 spectra with ages ranging from $1 \mathrm{Myr}$ to $2.5 \mathrm{Gyr}$. We allowed for a single extinction for all the base spectra that was modeled as a uniform dust screen with the extinction law by Cardelli et al. (1989).

The stellar spectral energy distribution at each spaxel was independently modelled for each cube. The spectral regions utilized in the fits were $3650-4060 \AA$ and the whole spectral range for the LR1 and LR2 gratings, respectively. All the main outputs for each spaxel were then reformatted in order to create three cubes per grating with the total, gaseous and modelled stellar emission. Additionally, the information associated to auxiliary properties such as stellar extinction in the $V$ band, the stellar velocity and velocity dispersion, the reduced $\chi^{2}$ and the absolute deviation of the fit (in \%) were reformatted and saved as a file suitable for manipulation with standard astronomical software. Hereafter, we will use both terms map and image to refer to these kind of files.

Representative examples of the achieved subtraction of the stellar component are shown in Fig. 2 which contains the observed, modeled and subtracted spectra for spaxels in the lowest surface brightness area, at the peak of emission in the continuum $\sharp 3$ and in the peak of emission for the ionized gas. The subtraction of the stellar continuum is satisfactory in all three cases. Note that given the spectral resolution of the FLAMES data (larger than the one of the base spectra), some fine structure (e.g. at $\sim 3930 \AA$ ) cannot be reproduced. However, this does not affect the recovery of the gaseous emission in which we are primarily interested.

\subsection{Line fitting and map creation}

As a first step, information from the relevant emission lines for each individual spaxel was obtained by fitting Gaussian

\footnotetext{
${ }^{3}$ Ages for the clusters associated to this knot were incorrectly quoted in our previous work. The ages estimated by Harris et al. (2004), for their clusters 3 and 5 were 8 and $11 \mathrm{Myr}$, respectively.

${ }^{4}$ http://starlight.ufsc.br/index .php?section=1

5 We assumed $12+\log (\mathrm{O} / \mathrm{H})_{\odot}=8.66$ (Asplund et al. 2004).
} 

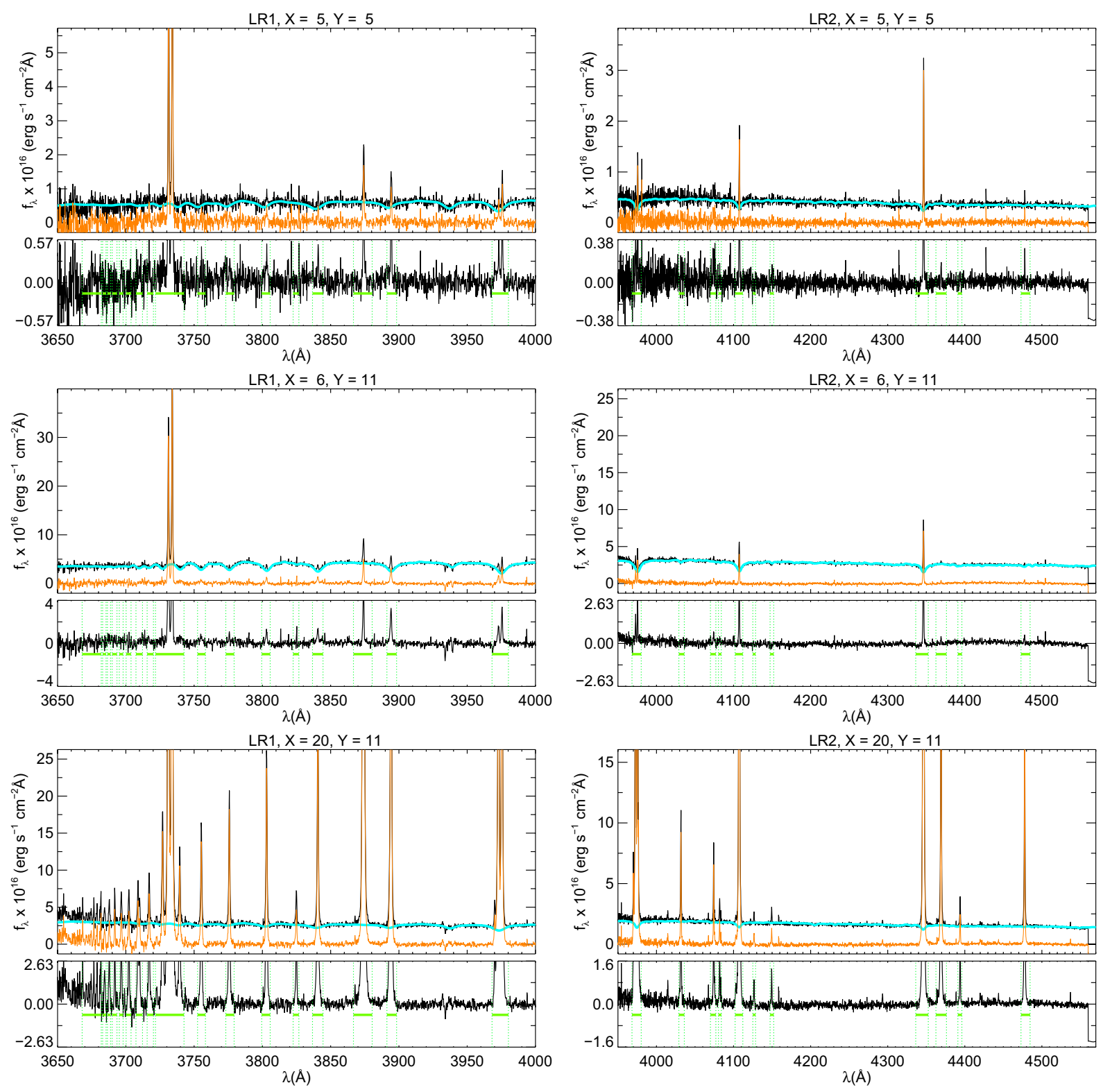

Fig. 2. Representative spectra to illustrate the modelling and subtraction of the underlying stellar population emission for the LR1 (left) and LR2 (right) data cubes. The selected spaxels show typical emission in the lowest surface brightness area (first row), the peak of continuum emission $\sharp 3$, associated to two relatively old clusters (second row), and the peak of emission in $\mathrm{H} \gamma$ (third row). Each individual graphic contains the observed, modelled and subtracted spectrum in black, cyan and orange, respectively. Below this main graphic there is an auxiliary one showing a zoom of the subtracted spectrum in black together with the spectral regions that were masked from the continuum fitting (green).

functions in a semi-automatic way using the IDL based routine MPFITEXPR (Markwardt 2009). Most of the lines (i.e. $\mathrm{H} \gamma$-H8, [O III] $\lambda 4363$, Не $1 \lambda 4009$, Не $1 \lambda 4026$, Не $1 \lambda 4388$, He I $\lambda 4471,\left[\mathrm{~S}_{\mathrm{II}}\right] \lambda 4074$ and [S II] $\lambda 4076$ ) were strong enough over most of the fov to be fitted independently. Exceptional cases were $[\mathrm{Ne} \mathrm{II}] \lambda 3967$ and $\mathrm{He}_{\mathrm{I}} \lambda 3965$ that were fitted simultaneously with $\mathrm{H} 7$, to assure a proper deblending of the emission lines and [O II] $\lambda 3727$ and $\left[\mathrm{O}_{\mathrm{II}}\right] \lambda 3729$ that were fitted simultaneously. For the rest of lines, the kinematic results for H7, present in both LR1 and LR2, were utilized as additional constraints. Several (strong) lines showed signs of asymmetries and/or multiple components in their profiles for a large number of spaxels. When possible, multi-component fits to these lines were also performed trying to assure a continuity in the derived physical (and chemical) properties between adjacent spaxels. In the present work, this multi-component fit will only be utilized in the discussion of the electron density structure. Finally, as we did with the fitting of the underlying stellar population, we used the derived quantities together with the position within the datacube for each spaxel to create a map.

On account of the different pointings between the 078.B-0043 and 383.B-0043 observing runs, an important issue for this analysis was assuring a proper match between maps for quantities derived from the LR3 and LR6 cubes and those of the LR1 and LR2. As a first guess we used the three main peaks in the continuum images as our reference to align the images. However, given the relatively small fov of the IFU, the extended nature of knots $\sharp 1$ and $\sharp 3$ and that knot $\sharp 2$ falls at the edge of the IFU in the LR1 and LR2 data, the derived offsets were not accurate enough. To refine this first guess, we created maps for ratios involving lines observed with the two different pointings (e.g. $[\mathrm{O}$ III $] \lambda 5007 /[\mathrm{O}$ III $] \lambda 4363$ ) using a grid of offsets centered on our 
first guess and in steps of 0.5 spaxels $(=0.26 \sim 1 / 4$ th seeing). We consider as final offset $[2.5 \mathrm{spa}, 5.0 \mathrm{spa}]\left(=\left[1^{\prime \prime} .3,2^{\prime \prime} 6\right]\right)$ which was the one providing the best continuity in adjacent spaxels for the variation of the line ratios. If the map of a given quantity was based on information from both runs, when possible, we independently carried out the calculations for 078.B-0043 and 383.B-0043. The offset was applied to the information associated to the 078.B-0043 run at the last step before carrying out the final calculation involving data from both runs. In this manner, the number of spatial interpolations was minimized.

\section{Results}

\subsection{Gaseous emission in selected apertures}

The main aim of the present work is to provide a full 2D characterization of the physical and chemical properties of the ionized gas in the central region of the galaxy. However, there are reasons to perform a more detailed analysis in specific regions. Firstly, we can define very precisely the aperture utilized to extract a given spectrum, avoiding the limitations associated to the positioning of a slit and assuring that the spectrum is spatially associated with a given feature of interest (e.g. the location of a star cluster). As an example, López-Sánchez et al. (2007) characterized the properties of our knot $\sharp 2$ (their UV-1). However, neither their positions HII-1 nor HII-2 sample the gas associated to the peak of emission in $\mathrm{H} \alpha$, but some emission associated to the GH IIR towards the west and south of the peak of emission. Secondly, a comparison of the measurements provided here, with those already existing in the literature serves as a sanity check which will reinforce the results obtained in the 2D mapping. Thirdly, most of the present methodologies used in the analysis of the physical and chemical properties of the ionized gas were developed to study complete $\mathrm{H}$ in regions. At present, the astronomical community is still in the process of understanding under which conditions these can be applied to small portions of them (e.g. Pérez-Montero et al. 2011; Ercolano et al. 2012). The areas analyzed in this section will be closer to this notion of complete $\mathrm{H}$ iI region than individual spaxels. Finally, by combining the signal of a collection of spaxels, a larger signalto-noise $(\mathrm{S} / \mathrm{N})$ ratio is achieved and fainter lines can be detected.

As we saw in Paper I, the stellar emission is dominated by three peaks of emission named as knots $\sharp 1, \sharp 2$ and $\sharp 3$ (see Table 3 of Paper I to establish the correspondence between this nomenclature and those of previous studies). Note that knot $\sharp 1$ is associated to the main GH IIR marked in Fig. 1. The spectra associated to them have been co-added and extracted in order to determine relative line intensities in their surrounding ionized gas.

The apertures utilized in the LR1 and LR2 data are outlined in Fig. 3. For the LR3 and LR6, we applied an offset of 2 and 5 spaxels in the $x$ and $y$ direction respectively. Since the offset between the two pointings was determined as [2.5 spa, $5.0 \mathrm{spa}$ ], this implies a small difference in the selected apertures from the old and the new data which introduces an extra uncertainty when comparing fluxes of lines belonging to a different set of data. In consequence, the line fluxes for the LR3 and the LR6 gratings were directly measured with respect to $\mathrm{H} \beta$ and $\mathrm{H} \alpha$ while those for the LR1 and LR2 gratings where measured with respect to $\mathrm{H} \gamma$ and $\mathrm{H} 7$, and then we assumed the theoretical Balmer line intensities obtained from Storey \& Hummer (1995) for Case B, $T_{\mathrm{e}}=10^{4} \mathrm{~K}$ and $n_{\mathrm{e}}=100 \mathrm{~cm}^{-3}$. Most of the lines were measured independently by fitting the emission to a single Gaussian profile using mpfit. Exceptionally, lines in the pairs H16 and $\mathrm{He}_{\mathrm{I}} \lambda 3705,[\mathrm{Fe}$ II] $\lambda 4414$ and [Fe II] $\lambda 4416$, and [Ar IV] $\lambda 4711$

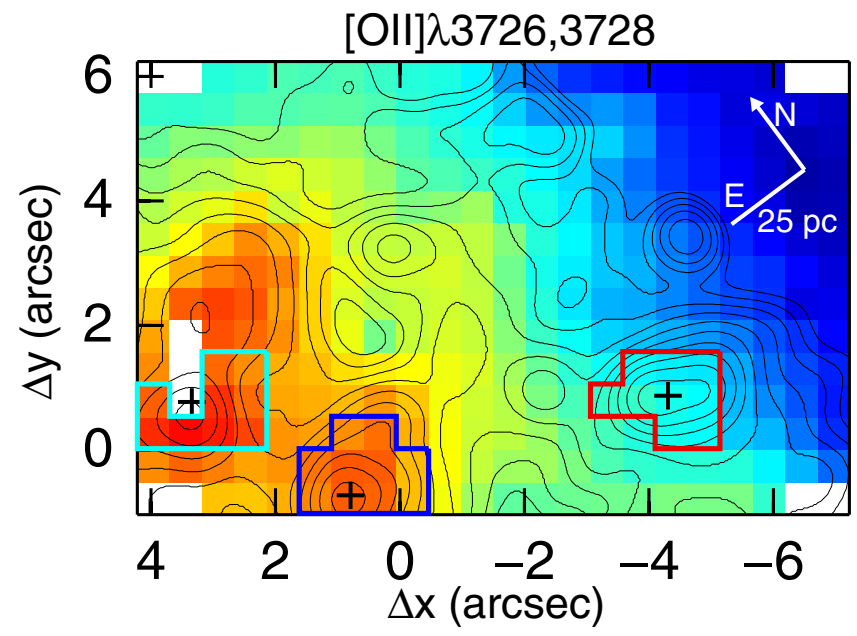

Fig. 3. Ionized gas distribution as traced by the [O II] emission line doublet. We have over-plotted contours corresponding to the HST-ACS images in the $F 435 W$ filter (programme 10609, P.I.: Vacca) convolved with a Gaussian of 0.8 to simulate the seeing at Paranal. The position of the three main peaks of continuum emission are marked with crosses. The map is presented in logarithmic scale in order to emphasize the relevant morphological features and cover a range of 1.4 dex. Flux units are arbitrary. Utilized apertures to extract the selected spectra have been outlined according to the following color code: cyan Knot $\sharp 1$; blue - Knot $\sharp 2$; red - Knot $\sharp 3$. Note the existence of three dead spaxels at $\sim\left[3{ }^{\prime \prime} 5,1^{\prime \prime} .0\right]$ as well as absence of signal in the spaxels at the four corners of the field of view. All of these spaxels will be marked hereafter by white rectangles. The scale and orientation are indicated.

and $\mathrm{He}$ I $\lambda 4713$ were simultaneously fitted due to their proximity in wavelength. Similarly, all the three lines Не I $\lambda 3965$, [Ne III] $\lambda 3967$ and $\mathrm{H} 7$ were fitted at once. Measurements for all detected lines are compiled in Tables 2 and 3.

\subsubsection{Physical conditions of the ionized gas}

Derived physical conditions for the ionized gas are listed in Table 4 . The values of reddening were derived from the measured $\mathrm{H} \alpha / \mathrm{H} \beta$ line ratio following the methodology described in Paper I. Electron temperature $\left(T_{\mathrm{e}}\right)$ and density $\left(n_{\mathrm{e}}\right)$ based on $\left[\mathrm{S}_{\mathrm{II}}\right],\left[\mathrm{O}_{\mathrm{II}}\right]$ and $\left[\mathrm{O}_{\mathrm{III}}\right]$ emission lines (and thus tracing different layers in the ionization structure) were derived using the expressions provided by García-Benito $(2009)^{6}$. We proceeded as follows: an initial $n_{\mathrm{e}}$ value of $100 \mathrm{~cm}^{-3}$ was assumed in order to obtain a first guess of the different $T_{\mathrm{e}}$ 's; then the resulting $T_{\mathrm{e}}\left(\left[\mathrm{O}_{\mathrm{II}}\right]\right)$ and $T_{\mathrm{e}}([\mathrm{S} \mathrm{II}])$ were used as input to obtain new estimates of $n_{\mathrm{e}}([\mathrm{O} \mathrm{II}])$ and $n_{\mathrm{e}}([\mathrm{S} \mathrm{II}])$ and the process was iterated until convergence. Typically one or two iterations were sufficient. These functions reproduce the predictions of the task temden, based on the fivel program (Shaw \& Dufour 1995) included in the IRAF package nebular. They used the same atomic coefficients as in Pérez-Montero \& Díaz (2003), except for $\mathrm{O}^{+}$for which they used the transition probabilities from Zeippen (1982) and the collision strengths from Pradhan (1976).

$T_{\mathrm{e}}([\mathrm{O} \mathrm{II}])$ was not independently derived from our data, since the $\left[\mathrm{O}_{\mathrm{II}}\right] \lambda \lambda 7320,7330$ doublet was not covered in these observations. Instead, we derived $T_{\mathrm{e}}([\mathrm{O} \mathrm{II}])$ from $T_{\mathrm{e}}([\mathrm{O} \mathrm{III}])$ and

\footnotetext{
6 http://www.iac.es/consolider-ingenio-gtc/index.php? option=com_content\&view=article\&id=223:qa-spatiallyresolved-study-of-ionized-regions-in-galaxies-atdifferent-scales\&catid=45: tesis\&Itemid=65
} 
Table 2. Observed fluxes for lines in the LR1 and the LR2 gratings with respect to $F(\mathrm{H} \beta)$.

\begin{tabular}{|c|c|c|c|c|c|}
\hline \multirow{2}{*}{$\begin{array}{l}\lambda_{0} \\
(\AA)\end{array}$} & \multirow[t]{2}{*}{ Ion } & \multirow[t]{2}{*}{$f(\lambda)$} & \multicolumn{3}{|c|}{$F(\lambda) / F(\mathrm{H} \beta)$} \\
\hline & & & Knot $\sharp 1$ & Knot $\sharp 2$ & Knot $\sharp 3$ \\
\hline 3669.47 & $\mathrm{HI}_{\mathrm{I}}$ & 0.268 & $0.17 \pm 0.05$ & $0.42 \pm 0.05$ & $\cdots$ \\
\hline 3671.48 & $\mathrm{HI}_{\mathrm{I}}$ & 0.268 & $0.24 \pm 0.05$ & $0.24 \pm 0.06$ & $\ldots$ \\
\hline 3673.76 & HI & 0.268 & $0.35 \pm 0.05$ & $0.46 \pm 0.06$ & $\ldots$ \\
\hline 3676.37 & $\mathrm{HI}_{\mathrm{I}}$ & 0.265 & $0.53 \pm 0.06$ & $0.57 \pm 0.07$ & $\ldots$ \\
\hline 3679.36 & $\mathrm{HI}_{\mathrm{I}}$ & 0.265 & $0.52 \pm 0.04$ & $0.69 \pm 0.11$ & $\ldots$ \\
\hline 3682.81 & $\mathrm{HI}_{\mathrm{I}}$ & 0.265 & $0.67 \pm 0.05$ & $0.77 \pm 0.08$ & $\ldots$ \\
\hline 3686.83 & $\mathrm{HI}_{\mathrm{I}}$ & 0.263 & $0.75 \pm 0.05$ & $0.99 \pm 0.08$ & $\ldots$ \\
\hline 3691.56 & $\mathrm{HI}_{\mathrm{I}}$ & 0.263 & $0.83 \pm 0.06$ & $0.91 \pm 0.06$ & $\ldots$ \\
\hline 3697.15 & $\mathrm{HI}_{\mathrm{I}}$ & 0.261 & $1.10 \pm 0.08$ & $0.91 \pm 0.06$ & $\ldots$ \\
\hline 3703.86 & $\mathrm{HI}_{\mathrm{I}}$ & 0.261 & $1.30 \pm 0.08$ & $1.25 \pm 0.06$ & $\ldots$ \\
\hline 3705.04 & $\mathrm{He} \mathrm{I}$ & 0.261 & $0.70 \pm 0.07$ & $0.57 \pm 0.06$ & $\ldots$ \\
\hline 3711.97 & $\mathrm{HI}_{\mathrm{I}}$ & 0.259 & $1.37 \pm 0.08$ & $1.71 \pm 0.09$ & $\ldots$ \\
\hline 3721.83 & [S III] & 0.257 & $2.95 \pm 0.16$ & $2.68 \pm 0.09$ & . \\
\hline 3726.03 & [O II] & 0.255 & $43.82 \pm 2.73$ & $75.36 \pm 2.05$ & $101.88 \pm 3.97$ \\
\hline 3728.82 & [O II] & 0.255 & $46.35 \pm 2.77$ & $93.87 \pm 2.61$ & $144.38 \pm 6.84$ \\
\hline 3734.17 & $\mathrm{HI}_{\mathrm{I}}$ & 0.255 & $1.99 \pm 0.14$ & $1.99 \pm 0.08$ & $1.26 \pm 0.20$ \\
\hline 3750.15 & $\mathrm{HI}_{\mathrm{I}}$ & 0.251 & $2.70 \pm 0.16$ & $2.80 \pm 0.10$ & $3.24 \pm 0.40$ \\
\hline 3770.63 & $\mathrm{HI}_{\mathrm{I}}$ & 0.247 & $3.34 \pm 0.18$ & $3.54 \pm 0.09$ & $2.51 \pm 0.32$ \\
\hline 3797.90 & $\mathrm{HI}_{\mathrm{I}}$ & 0.241 & $4.59 \pm 0.25$ & $4.69 \pm 0.12$ & $4.56 \pm 0.33$ \\
\hline 3819.61 & $\mathrm{He}$ I & 0.237 & $0.86 \pm 0.06$ & $0.88 \pm 0.04$ & $0.65 \pm 0.20$ \\
\hline 3835.39 & $\mathrm{HI}_{\mathrm{I}}$ & 0.235 & $6.61 \pm 0.35$ & $6.74 \pm 0.15$ & $6.65 \pm 0.48$ \\
\hline 3868.75 & [Ne III] & 0.227 & $48.87 \pm 3.10$ & $31.73 \pm 0.93$ & $22.34 \pm 0.89$ \\
\hline 3889.05 & $\mathrm{HI}+\mathrm{He} \mathrm{I}$ & 0.223 & $16.52 \pm 0.90$ & $18.11 \pm 0.43$ & $15.91 \pm 0.67$ \\
\hline 3964.73 & He I & 0.209 & $0.57 \pm 0.35$ & $0.59 \pm 0.19$ & $0.77 \pm 0.45$ \\
\hline 3967.46 & [Ne III] & 0.207 & $15.88 \pm 0.82$ & $10.38 \pm 0.31$ & $8.77 \pm 0.59$ \\
\hline 3970.07 & $\mathrm{HI}_{\mathrm{I}}$ & 0.207 & $15.80 \pm 0.82$ & $15.80 \pm 0.38$ & $15.80 \pm 0.72$ \\
\hline 4009.22 & $\mathrm{He}_{\mathrm{I}}$ & 0.198 & $0.20 \pm 0.03^{*}$ & & \\
\hline 4026.21 & $\mathrm{He}_{\mathrm{I}}$ & 0.194 & $1.73 \pm 0.09$ & $1.69 \pm 0.05$ & $0.81 \pm 0.19$ \\
\hline 4068.60 & {$\left[\mathrm{~S}_{\text {II] }}\right]$} & 0.187 & $1.40 \pm 0.08$ & $1.41 \pm 0.05$ & $3.28 \pm 0.24$ \\
\hline 4076.35 & {$\left[\mathrm{~S}_{\text {II] }}\right]$} & 0.184 & $0.46 \pm 0.03$ & $0.52 \pm 0.03$ & $1.25 \pm 0.16$ \\
\hline 4097.26 & $\mathrm{O}_{\text {II }}$ & 0.180 & $0.05 \pm 0.01$ & & \\
\hline 4101.74 & $\mathrm{HI}_{\mathrm{I}}$ & 0.180 & $25.79 \pm 1.37$ & $25.42 \pm 0.58$ & $25.19 \pm 0.89$ \\
\hline 4120.82 & $\mathrm{He}_{\mathrm{I}}$ & 0.176 & $0.20 \pm 0.02$ & $0.09 \pm 0.03$ & $\ldots$ \\
\hline 4143.76 & He I & 0.172 & $0.23 \pm 0.02$ & $0.16 \pm 0.02$ & $\ldots$ \\
\hline 4243.97 & {$[\mathrm{Fe}$ II] } & 0.153 & $0.03 \pm 0.01$ & $0.05 \pm 0.01$ & . \\
\hline 4267.15 & $\mathrm{C}_{\text {II }}$ & 0.147 & $0.06 \pm 0.01$ & $0.10 \pm 0.01$ & \\
\hline 4287.40 & {$[\mathrm{Fe}$ II] } & 0.144 & $0.12 \pm 0.01$ & $0.24 \pm 0.02$ & $1.23 \pm 0.15$ \\
\hline 4340.47 & $\mathrm{HI}_{\mathrm{I}}$ & 0.133 & $46.60 \pm 2.87$ & $46.60 \pm 1.12$ & $46.60 \pm 1.71$ \\
\hline 4359.34 & {$[\mathrm{Fe}$ II] } & 0.129 & $0.06 \pm 0.01$ & $0.13 \pm 0.01$ & $0.53 \pm 0.10$ \\
\hline 4363.21 & [O III] & 0.129 & $7.77 \pm 0.39$ & $3.70 \pm 0.08$ & $2.64 \pm 0.21$ \\
\hline 4368.25 & OI & 0.127 & $0.03 \pm 0.01$ & & $\ldots$ \\
\hline 4387.93 & He I & 0.122 & $0.49 \pm 0.03$ & $0.45 \pm 0.02$ & $\ldots$ \\
\hline 4413.78 & {$[\mathrm{Fe}$ II] } & 0.118 & $0.07 \pm 0.01$ & $0.01 \pm 0.01$ & $\ldots$ \\
\hline 4416.27 & {$[\mathrm{Fe}$ II] } & 0.115 & $0.04 \pm 0.01$ & $0.03 \pm 0.01$ & \\
\hline 4437.55 & He I & 0.110 & $4.48 \pm 0.25$ & . & $\ldots$ \\
\hline 4452.11 & {$[\mathrm{Fe}$ II] } & 0.108 & $0.13 \pm 0.01$ & & \\
\hline 4471.48 & $\mathrm{He}_{\mathrm{I}}$ & 0.103 & $0.08 \pm 0.01$ & $0.19 \pm 0.01$ & $0.36 \pm 0.18$ \\
\hline
\end{tabular}

Notes. See text for details of the scaling of the spectra. ${ }^{(*)}$ This line presented a "secondary peak" towards the blue which correspond to a weak [Fe III] line at $\lambda 4008$. At the resolution of the present data a proper deblending of the lines was not feasible.

$n_{\mathrm{e}}([\mathrm{O}$ II $])$ using the models presented by Pérez-Montero \& Díaz (2003). As an improvement to former relations based on modelling (e.g. Stasińska 1990), these take into account dependencies on $n_{\mathrm{e}}$. At low densities, for a given $T_{\mathrm{e}}([\mathrm{O} \mathrm{III}])$, models by Pérez-Montero \& Díaz (2003) predict only slightly (i.e. $\$ 2 \%$ ) lower temperatures than those by Stasińska (1990) while in the densest zones of NGC 5253 (e.g. our knot $\sharp 1$ ), the estimated $T_{\mathrm{e}}([\mathrm{O} \mathrm{II}])$ can be up to $\sim 17 \%$ smaller. For $n_{\mathrm{e}}([\mathrm{Fe} \mathrm{III}])$, we used the line ratios tabulated by Keenan et al. (2001), assuming $T_{\mathrm{e}}=12000 \mathrm{~K}$. Note that for the emission lines utilized, the dependence of the derived $n_{\mathrm{e}}$ on the assumed temperature is negligible. Finally, $n_{\mathrm{e}}([\mathrm{Ar} \mathrm{IV}])$ was derived using directly temden and assuming $T_{\mathrm{e}}([$ Ar IV $])=T_{\mathrm{e}}([\mathrm{O} \mathrm{III}])$.
Table 3. Observed fluxes for lines in the LR3 and the LR6 gratings with respect to $\mathrm{F}(\mathrm{H} \beta)$.

\begin{tabular}{|c|c|c|c|c|c|}
\hline \multirow{2}{*}{$\begin{array}{l}\lambda_{0} \\
(\AA)\end{array}$} & \multirow[t]{2}{*}{ Ion } & \multirow[t]{2}{*}{$f(\lambda)$} & \multicolumn{3}{|c|}{$F(\lambda) / F(\mathrm{H} \beta)$} \\
\hline & & & Knot $\sharp 1$ & Knot $\sharp 2$ & Knot $\sharp 3$ \\
\hline 4562.60 & $\left.\mathrm{Mg}_{\mathrm{I}}\right]$ & 0.080 & $0.11 \pm 0.01$ & $0.19 \pm 0.01$ & $0.86 \pm 0.19$ \\
\hline 4571.00 & $\left.\mathrm{Mg}_{\mathrm{I}}\right]$ & 0.078 & $0.12 \pm 0.01$ & $0.16 \pm 0.01$ & $0.74 \pm 0.17$ \\
\hline 4649.13 & $\mathrm{O}_{\text {II }}$ & 0.057 & $0.08 \pm 0.01$ & & \\
\hline 4658.10 & {$\left[\mathrm{Fe}_{\mathrm{III}}\right]$} & 0.054 & $0.96 \pm 0.05$ & $0.80 \pm 0.03$ & $2.34 \pm 0.19$ \\
\hline 4701.53 & {$[\mathrm{Fe}$ III] } & 0.043 & $0.27 \pm 0.01$ & $0.23 \pm 0.01$ & $0.84 \pm 0.19$ \\
\hline 4711.37 & [Ar IV] & 0.041 & $1.17 \pm 0.06$ & $0.19 \pm 0.00$ & $0.57 \pm 0.02$ \\
\hline 4713.14 & $\mathrm{He}_{\mathrm{I}}$ & 0.041 & $0.65 \pm 0.05$ & $0.41 \pm 0.01$ & $0.19 \pm 0.16$ \\
\hline 4740.16 & [Ar IV] & 0.032 & $1.18 \pm 0.05$ & $0.17 \pm 0.01$ & \\
\hline 4754.83 & {$[\mathrm{Fe}$ III] } & 0.030 & $0.19 \pm 0.01$ & $0.18 \pm 0.01$ & \\
\hline 4769.60 & {$[\mathrm{Fe}$ III] } & 0.024 & $0.10 \pm 0.01$ & $0.10 \pm 0.01$ & $\ldots$ \\
\hline 4814.55 & {$[\mathrm{Fe}$ II $]$} & 0.013 & $0.06 \pm 0.01$ & & \\
\hline 4861.33 & $\mathrm{HI}_{\mathrm{I}}$ & 0.000 & $100.00 \pm 4.36$ & $100.00 \pm 1.82$ & $100.00 \pm 2.51$ \\
\hline 4881.00 & {$[\mathrm{Fe}$ III] } & -0.005 & $0.37 \pm 0.02$ & $0.25 \pm 0.02$ & $\ldots$ \\
\hline 4921.93 & He I & -0.016 & $1.14 \pm 0.05$ & $1.03 \pm 0.03$ & \\
\hline 4931.32 & [O III] & -0.019 & $0.16 \pm 0.01$ & $0.16 \pm 0.02$ & \\
\hline 4958.91 & [O III] & -0.027 & $232.56 \pm 10.88$ & $162.83 \pm 3.25$ & $114.29 \pm 3.25$ \\
\hline 4985.90 & {$[\mathrm{Fe} \mathrm{III}]$} & -0.032 & $0.47 \pm 0.03$ & $0.64 \pm 0.03$ & $2.78 \pm 0.26$ \\
\hline 5006.84 & [O III] & -0.040 & $732.56 \pm 34.05$ & $508.85 \pm 10.23$ & $358.10 \pm 10.25$ \\
\hline 5015.68 & $\mathrm{He}_{\mathrm{I}}$ & -0.043 & $1.87 \pm 0.09$ & $2.19 \pm 0.06$ & $2.11 \pm 0.25$ \\
\hline 5041.03 & SI II & -0.049 & $0.24 \pm 0.02$ & $0.16 \pm 0.01$ & $\ldots$ \\
\hline 5055.98 & SI II & -0.055 & $\ldots$ & $0.16 \pm 0.01$ & $\ldots$ \\
\hline 6548.03 & {$[\mathrm{~N}$ II $]$} & -0.312 & $10.70 \pm 0.46$ & $5.88 \pm 0.16$ & $11.33 \pm 0.29$ \\
\hline 6562.82 & $\mathrm{HI}_{\mathrm{I}}$ & -0.314 & $400.00 \pm 16.86$ & $391.15 \pm 7.37$ & $415.24 \pm 10.18$ \\
\hline 6583.41 & {$\left[\mathrm{~N}_{\mathrm{II}}\right]$} & -0.316 & $32.67 \pm 1.39$ & $17.96 \pm 0.49$ & $35.05 \pm 0.80$ \\
\hline 6678.15 & $\mathrm{He}_{\mathrm{I}}$ & -0.329 & $4.55 \pm 0.20$ & $4.20 \pm 0.08$ & 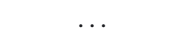 \\
\hline 6716.47 & {$[\mathrm{~S}$ II $]$} & -0.332 & $13.95 \pm 0.60$ & $20.53 \pm 0.41$ & $55.24 \pm 1.32$ \\
\hline 6730.85 & [S II] & -0.335 & $12.91 \pm 0.55$ & $16.55 \pm 0.34$ & $40.76 \pm 0.98$ \\
\hline 7002.23 & $\mathrm{O}_{\mathrm{I}}$ & -0.365 & $0.09 \pm 0.01$ & $0.10 \pm 0.01$ & \\
\hline 7065.28 & $\mathrm{He}_{\mathrm{I}}$ & -0.375 & $7.73 \pm 0.30$ & $3.85 \pm 0.08$ & $2.83 \pm 0.19$ \\
\hline 7135.78 & [Ar III] & -0.384 & $16.34 \pm 0.73$ & $12.65 \pm 0.26$ & $10.67 \pm 0.30$ \\
\hline
\end{tabular}

Notes. See text for details of the scaling of the spectra.

Table 4. Physical properties of the ionized gas.

\begin{tabular}{lccc}
\hline \hline & Knot $\sharp 1$ & Knot $\sharp 2$ & Knot $\sharp 3$ \\
\hline$E(B-V)$ & $0.31 \pm 0.04$ & $0.29 \pm 0.02$ & $0.35 \pm 0.04$ \\
\hline$n_{\mathrm{e}}\left(\mathrm{cm}^{-3}\right)\left(\left[\mathrm{O}_{\mathrm{II}}\right]\right)$ & $310_{-140}^{+150}$ & $140_{-50}^{+50}$ & $30_{-60}^{+100}$ \\
$n_{\mathrm{e}}\left(\mathrm{cm}^{-3}\right)\left(\left[\mathrm{S}_{\mathrm{II}}\right]\right)$ & $385_{-120}^{+150}$ & $170_{-30}^{+35}$ & $70_{-25}^{+30}$ \\
$n_{\mathrm{e}}\left(\mathrm{cm}^{-3}\right)([\mathrm{Ar} \mathrm{IV}])$ & $4600_{-1000}^{+1200}$ & $2800_{-900}^{+1800}$ & $\ldots$ \\
$n_{\mathrm{e}}\left(\mathrm{cm}^{-3}\right)([\mathrm{Fe}$ III $])$ & $270_{-30}^{+40}$ & $155_{-15}^{+20}$ & $95_{-20}^{+25}$ \\
\hline$T_{\mathrm{e}}(\mathrm{K})\left(\left[\mathrm{O}_{\mathrm{III}}\right]\right)$ & $11570_{-350}^{+420}$ & $10280_{-120}^{+130}$ & $10320_{-300}^{+350}$ \\
$T_{\mathrm{e}}(\mathrm{K})([\mathrm{O}$ II $])$ & 11360 & 10470 & 10500 \\
$T_{\mathrm{e}}(\mathrm{K})\left(\left[\mathrm{S}_{\mathrm{II}}\right]\right)$ & $9700_{-1300}^{+1700}$ & $8700_{-440}^{+490}$ & $8900_{-680}^{+870}$ \\
\hline
\end{tabular}

Notes. - Ratios for $n_{\mathrm{e}}$ : [O $\left.\mathrm{II}\right]: \quad\left[\mathrm{O}_{\text {II }}\right] \lambda 3727 /\left[\mathrm{O}_{\text {II }}\right] \lambda 3729 ; \quad\left[\mathrm{S}_{\text {II }}\right]$ : [S II] $26717 /[\mathrm{S}$ II] $6731 ; \quad$ [Ar Iv]: [Ar IV] $\lambda 4711 /[\mathrm{Ar}$ Iv] $\lambda 4740 ; \quad$ [Fe III]: $\left[\mathrm{Fe}_{\text {III }}\right] \lambda 4986 /\left[\mathrm{Fe}\right.$ III] $\lambda 4658$. - Ratios for $T_{\mathrm{e}}$ : [O III] $[$ [O III] $] \lambda \lambda 4959,5007 /$ [O III] $\lambda 4363 ;$ [S II]: [S II] $\lambda \lambda 6716,6731 /\left[\mathrm{S}_{\text {II }}\right] \lambda 4069,4076$. Note that $T_{\mathrm{e}}\left(\left[\mathrm{O}_{\mathrm{II}}\right]\right)$ was not obtained directly from measurements of $\left[\mathrm{O}_{\mathrm{II}}\right]$ lines. Instead, it was derived from $T_{\mathrm{e}}\left(\left[\mathrm{O}_{\mathrm{III}}\right]\right)$ and $n_{\mathrm{e}}\left(\left[\mathrm{O}_{\mathrm{II}}\right]\right)$ according to the expressions provided in García-Benito (2009).

Derived $T_{\mathrm{e}}$ and $n_{\mathrm{e}}$ for our knots $\sharp 1$ and $\sharp 2$ agree well within the uncertainties with the values reported by Sidoli (2010) for his apertures $\mathrm{A}$ and $\mathrm{B}$, respectively. Additional measurements for $T_{\mathrm{e}}$ and $n_{\mathrm{e}}$ in knot $\sharp 2$ are provided by López-Sánchez et al. (2007), Kobulnicky et al. (1997) and Guseva et al. (2011). While our derived $T_{\mathrm{e}}$ 's are in relatively good agreement, with $T_{\mathrm{e}}([\mathrm{O} \mathrm{III}])$ and $T_{\mathrm{e}}$ ([S II]) being slightly lower and larger respectively than the values reported in these works, there is a discrepancy between 
Table 5. Ionic chemical abundances of the ionized gas.

\begin{tabular}{lccc}
\hline \hline & Knot $\sharp 1$ & Knot $\sharp 2$ & Knot $\sharp 3$ \\
\hline $12+\log \left(\mathrm{O}^{+} / \mathrm{H}^{+}\right)$ & $7.33 \pm 0.10$ & $7.73 \pm 0.04$ & $7.88 \pm 0.09$ \\
$12+\log \left(\mathrm{O}^{2+} / \mathrm{H}^{+}\right)$ & $8.18 \pm 0.09$ & $8.17 \pm 0.03$ & $8.03 \pm 0.07$ \\
$12+\log (\mathrm{O} / \mathrm{H})$ & $8.24 \pm 0.09$ & $8.32 \pm 0.04$ & $8.26 \pm 0.08$ \\
\hline $12+\log \left(\mathrm{S}^{+} / \mathrm{H}^{+}\right)$ & $5.71 \pm 0.07$ & $5.96 \pm 0.03$ & $6.31 \pm 0.05$ \\
$\log \left(\mathrm{S}^{+} / \mathrm{O}^{+}\right)$ & $-1.62 \pm 0.17$ & $-1.77 \pm 0.07$ & $-1.57 \pm 0.14$ \\
\hline $12+\log \left(\mathrm{N}^{+} / \mathrm{H}^{+}\right)$ & $6.52 \pm 0.07$ & $6.36 \pm 0.03$ & $6.62 \pm 0.06$ \\
$12+\log (\mathrm{N} / \mathrm{H})$ & $7.43 \pm 0.07$ & $6.94 \pm 0.03$ & $7.00 \pm 0.06$ \\
$\mathrm{ICF}\left(\mathrm{N}^{+}\right)$ & 8.04 & 3.84 & 2.40 \\
$\log (\mathrm{N} / \mathrm{O})$ & $-0.81 \pm 0.25$ & $-1.37 \pm 0.11$ & $-1.26 \pm 0.22$ \\
\hline $12+\log \left(\mathrm{Ne} \mathrm{e}^{2+} / \mathrm{H}^{+}\right)$ & $7.49 \pm 0.10$ & $7.50 \pm 0.04$ & $7.34 \pm 0.09$ \\
$\mathrm{ICF}(\mathrm{Ne})$ & 1.14 & 1.35 & 1.71 \\
$12+\log (\mathrm{Ne} / \mathrm{H})$ & $7.55 \pm 0.10$ & $7.63 \pm 0.04$ & $7.57 \pm 0.09$ \\
$\log (\mathrm{Ne} / \mathrm{O})$ & $-0.69 \pm 0.28$ & $-0.69 \pm 0.12$ & $-0.69 \pm 0.25$ \\
\hline $12+\log \left(\mathrm{Ar}{ }^{2+} / \mathrm{H}^{+}\right)$ & $5.89 \pm 0.07$ & $5.90 \pm 0.03$ & $5.80 \pm 0.05$ \\
$12+\log \left(\mathrm{Ar}{ }^{3+} / \mathrm{H}^{+}\right)$ & $4.84 \pm 0.08$ & $4.15 \pm 0.05$ & $\ldots$ \\
$\mathrm{ICF}(\mathrm{Ar})^{*}$ & $1.02 \pm 0.04$ & $1.07 \pm 0.05$ & $1.45 \pm 1.91$ \\
$12+\log (\mathrm{Ar} / \mathrm{H})$ & $5.90 \pm 0.22$ & $5.93 \pm 0.12$ & $5.96 \pm 1.45$ \\
$\log (\mathrm{Ar} / \mathrm{O})$ & $-2.34 \pm 0.30$ & $-2.38 \pm 0.16$ & $-2.30 \pm 1.53$ \\
\hline $12+\log \left(\mathrm{Fe}{ }^{2+} / \mathrm{H}^{+}\right)$ & $5.58 \pm 0.09$ & $5.66 \pm 0.04$ & $6.12 \pm 0.09$ \\
\hline
\end{tabular}

Notes. ${ }^{(*)} \mathrm{ICF}\left(\mathrm{Ar}^{2+}\right)$ for knot $\sharp 3$.

the different measurements of $n_{\mathrm{e}}$ in the literature. A comparison of our $n_{\mathrm{e}}([\mathrm{S} \mathrm{II}])$ for knot $\sharp 1$ and the values reported by Guseva et al. (2011) for their apertures C1 and P2 shows a discrepancy between the reported values. Since $n_{\mathrm{e}}$ varies a lot in this area (a factor of $\sim 2-3$ on scales of $\sim 0{ }^{\prime} 5$, see Fig. 9 in Sect. 4.1), the precise definition of the aperture (i.e. size, position and shape) in each case seems the most plausible explanation for this discrepancy. The value of $n_{\mathrm{e}} \sim 150 \mathrm{~cm}^{-3}$ in knot $\sharp 2$ is consistent, within the errors, with being below the low density limit as reported by Kobulnicky et al. (1997). However, it is a factor $\sim 2$ lower than the values reported by López-Sánchez et al. (2007). While estimated uncertainties could account for the differences in $n_{\mathrm{e}}\left(\left[\mathrm{S}_{\mathrm{II}}\right]\right)$, one must resort to other causes, such as differences in the definition of the aperture, to explain the discrepancies in $n_{\mathrm{e}}([\mathrm{O} \mathrm{II}])$.

\subsubsection{Chemical abundances}

Abundances for the different ionic species are listed in Table 5 and were derived using the relations provided in Appendix B.2 of García-Benito (2009). These are appropriate fittings to the results of the IRAF task ionic (Shaw \& Dufour 1995), based on the 5-level atom program developed by De Robertis et al. (1987), and follow the functional form given by Pagel et al. (1992). For the abundances of neon, argon, iron and $\mathrm{O}^{++}$, we utilized $T_{\mathrm{e}}([\mathrm{O} \mathrm{III}])$. For $\mathrm{O}^{+}$and nitrogen, we utilized $T_{\mathrm{e}}\left(\left[\mathrm{O}_{\mathrm{II}}\right]\right)$, derived from $T_{\mathrm{e}}([\mathrm{O} \mathrm{III}])$ and $n_{\mathrm{e}}\left(\left[\mathrm{O}_{\mathrm{II}}\right]\right)$. Finally, for sulfur, we took $T_{\mathrm{e}}([\mathrm{S} \mathrm{II}])$. Differences in abundances due to the assumed $T_{\mathrm{e}}$ are discussed below. To derive the total abundances, unseen ionization stages of a given element were taken into account by including the appropriate ionization factor (ICF) for each species when necessary, following the prescriptions provided by Kingsburgh \& Barlow (1994) for all the elements but argon. For this element, we utilized those provided by Izotov et al. (1994).

Oxygen: we assumed $\mathrm{O} / \mathrm{H}=\left(\mathrm{O}^{+}+\mathrm{O}^{2+}\right) / \mathrm{H}^{+}$. The derived values are in good agreement with those provided in the literature using long-slit data (e.g. Guseva et al. 2011; Sidoli
2010; López-Sánchez et al. 2007; Kobulnicky et al. 1997). The largest differences are for knot $\sharp 1$ that shows abundances $\sim 0.1$ dex higher than those measured by Guseva et al. (2011). This is however within the uncertainties.

Sulfur: derived values are typically $\sim 0.2$ dex larger than those provided by López-Sánchez et al. (2007). Differences can be attributed to the adopted $T_{\mathrm{e}}$. López-Sánchez et al. (2007) use the average of $T_{\mathrm{e}}\left(\left[\mathrm{S}_{\mathrm{II}}\right]\right), T_{\mathrm{e}}\left(\left[\mathrm{O}_{\mathrm{II}}\right]\right)$, and $T_{\mathrm{e}}\left(\left[\mathrm{N}_{\mathrm{II}}\right]\right)$ that can be $\sim 2000 \mathrm{~K}$ larger than $T_{\mathrm{e}}$ ([S II $\left.]\right)$. If $T_{\mathrm{e}}\left(\left[\mathrm{O}_{\mathrm{II}}\right]\right)$, derived from the $n_{\mathrm{e}}\left(\left[\mathrm{O}_{\mathrm{II}}\right]\right)$ and $T_{\mathrm{e}}([\mathrm{O} \mathrm{III}])$, were utilized, our abundances would become typically $\sim 0.15$ dex smaller in agreement with the values reported by these authors. A similar effect is observed when comparing with the results reported by Sidoli (2010) who utilized $T_{\mathrm{e}}([\mathrm{O} \mathrm{III}])$ to derive all the abundances and those reported by Guseva et al. (2011) who used a $T_{\mathrm{e}}([\mathrm{O}$ II] $) \sim 1500-2000 \mathrm{~K}$ larger than the $T_{\mathrm{e}}([\mathrm{S}$ II $])$ utilized here.

Nitrogen: we assumed $\mathrm{N} / \mathrm{O}=\mathrm{N}^{+} / \mathrm{O}^{+}$, which is an accurate approximation to about $\pm 20 \%$ for nebulae with metallicities smaller than that in the LMC (Garnett 1990). In agreement with previous work, while knots $\sharp 2$ and $\sharp 3$ present relative abundances of nitrogen within the range expected for galaxies at this metallicity (see e.g. Mollá et al. 2006), the aperture associated to knot $\sharp 1$ present a clear excess in nitrogen. Interestingly, although consistent with the N/O expected for galaxies at this metallicity, knot $\sharp 3$ has a slightly larger abundance than knot $\sharp 2$.

Neon: since the ionization structure is similar to the one of the oxygen, we assumed $\mathrm{Ne}^{2+} / \mathrm{Ne}=\mathrm{O}^{2+} / \mathrm{O}$. Reported values agree within the errors with previous measurements in the literature (Guseva et al. 2011; López-Sánchez et al. 2007; Kobulnicky et al. 1997).

Argon: total argon abundance was derived using the ICFs based on the expressions provided by Izotov et al. (1994). Derived ratios are within the range of those previously published (Guseva et al. 2011; López-Sánchez et al. 2007; Sidoli 2010) and consistent with a homogeneous $\mathrm{Ar} / \mathrm{O}$ ratio across the face of the galaxy.

Iron: reported values for $\mathrm{Fe}^{2}+$ agree with those given by López-Sánchez et al. (2007).

To recap, abundances of the different ionic species in knot $\sharp 1$ and knot $\sharp 2$ agree in general with those previously reported (Kobulnicky et al. 1997; López-Sánchez et al. 2007; Sidoli 2010). Abundances in knot $\sharp 3$ (not reported so far) are similar to those in knot $\sharp 1$ and knot $\sharp 2$. With the exception of the relative abundance for N/O in knot $\sharp 1$ and maybe knot $\sharp 3$, no chemical species seems overabundant in any of the selected apertures. This is in agreement with the relative enrichment in nitrogen previously widely reported.

\subsection{Mapping electron density and temperature tracers}

In Paper I, we found an $n_{\mathrm{e}}\left(\left[\mathrm{S}_{\mathrm{II}}\right]\right)$ gradient declining from the peak of emission in $\mathrm{H} \alpha$ outwards and traced the gas at the highest densities by means of the $n_{\mathrm{e}}([\mathrm{Ar} \mathrm{IV}])$. Also, our multi-component analysis of the $\left[\mathrm{S}_{\mathrm{II}}\right]$ line profiles tentatively suggested similar densities over the whole face of the main $\mathrm{H}$ in region for the broad component, while the narrow component presented somewhat lower (higher) ratios towards the NW (SE) part of the region. Given the restricted spectral range utilized in that work, the $T_{\mathrm{e}}$ structure could not be derived.

Here, we present maps for two additional tracers of $n_{\mathrm{e}}$ : $\left[\mathrm{O}_{\text {II }}\right] \lambda 3726 /\left[\mathrm{O}_{\mathrm{II}}\right] \lambda 3729$ and [Fe III] $] \lambda 4986 /[\mathrm{Fe}$ III] $] \lambda 4658$. In comparison to $[\mathrm{S}$ II $] \lambda 6717 /\left[\mathrm{S}_{\mathrm{II}}\right] \lambda 6731$, the first ratio is sensitive to a slightly larger range of densities, tracing to lower $n_{\mathrm{e}}$ (Osterbrock $\&$ Ferland 2006). Moreover, the [O II] lines are stronger than 

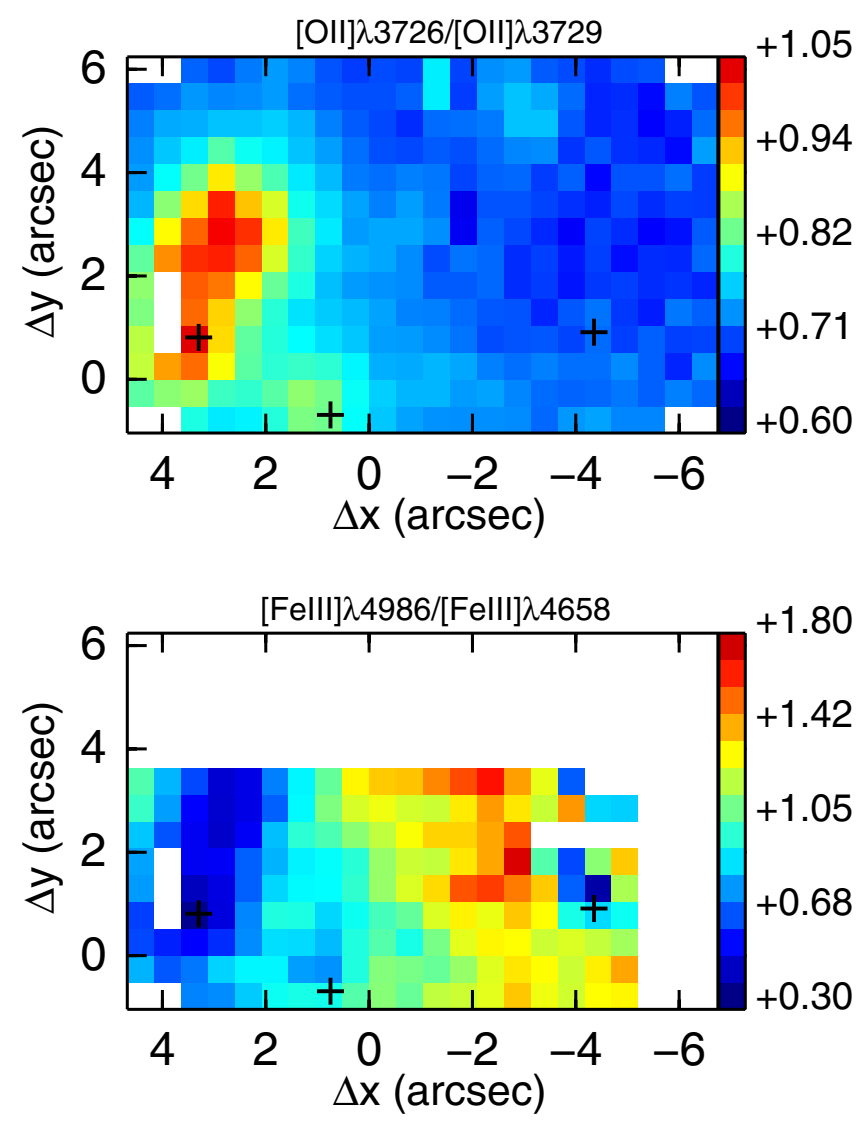

Fig. 4. Maps for the two line ratios sensitive to the electron density. The position of the three main peaks of continuum emission are shown as crosses for reference.

those of [S II]. Therefore, we will be able to more systematically discuss the differences in $n_{\mathrm{e}}$ structure between different kinematic components, as suggested by the $\left[\mathrm{S}_{\mathrm{II}}\right]$ line ratio. The [Fe III] ratio is sensitive to an even larger range of densities, from $\sim 10^{2} \mathrm{~cm}^{-3}$ to $\lesssim 10^{7} \mathrm{~cm}^{-3}$ (Keenan et al. 2001) and has already been used with success in similar works to this one to reveal very high density locations (James et al. 2009). Also, we will introduce for the first time maps for two tracers of $T_{\mathrm{e}}$ in this galaxy: [O $\left.\mathrm{mI}\right] \lambda \lambda 4959,5007 /[\mathrm{O}$ III] $] \lambda 4363$ and $[\mathrm{S}$ II] $] \lambda 6717,6731 /[\mathrm{S}$ II] $] \lambda \lambda 4069,4076$. By comparing the maps in $n_{\mathrm{e}}$ and $T_{\mathrm{e}}$ according to the different tracers, we will be able to see how the physical properties of the gas are structured in the different ionization layers (see Sect. 4).

The maps for our new $n_{\mathrm{e}}$ tracers are presented in Fig. 4. For that involving the $[\mathrm{O}$ I] emission lines, large values, associated to relatively large $n_{\mathrm{e}}$, are found in the main GH IIR. There is a secondary maximum at the location of knot $\$ 2$ and ratios typical of low densities elsewhere. The map for the [Fe III] line ratios presents the inverse tendencies: the maximum of [O II] line ratio corresponds to the minimum of the [Fe III] line ratio. The two overall structures are similar to the one depicted by the $\left[\mathrm{S}_{\mathrm{II}}\right]$ line ratios (Paper I).

More interestingly, Fig. 5 presents the maps for the line ratios tracing the $T_{\mathrm{e}}$. Both of them display similar structure, with the absolute minimum at the location of the two SSCs (i.e. knot $\sharp 1$ ), corresponding to the highest $T_{\mathrm{e}}$, ratios remaining relatively low in the GH IIR and then tending to higher values in the rest of the fov. As with the selected apertures in Sect. 3.1.1, on each individual spaxel the predicted $T_{\mathrm{e}}$ from the [O $\left.\mathrm{O}_{\mathrm{III}}\right]$ line ratio differs
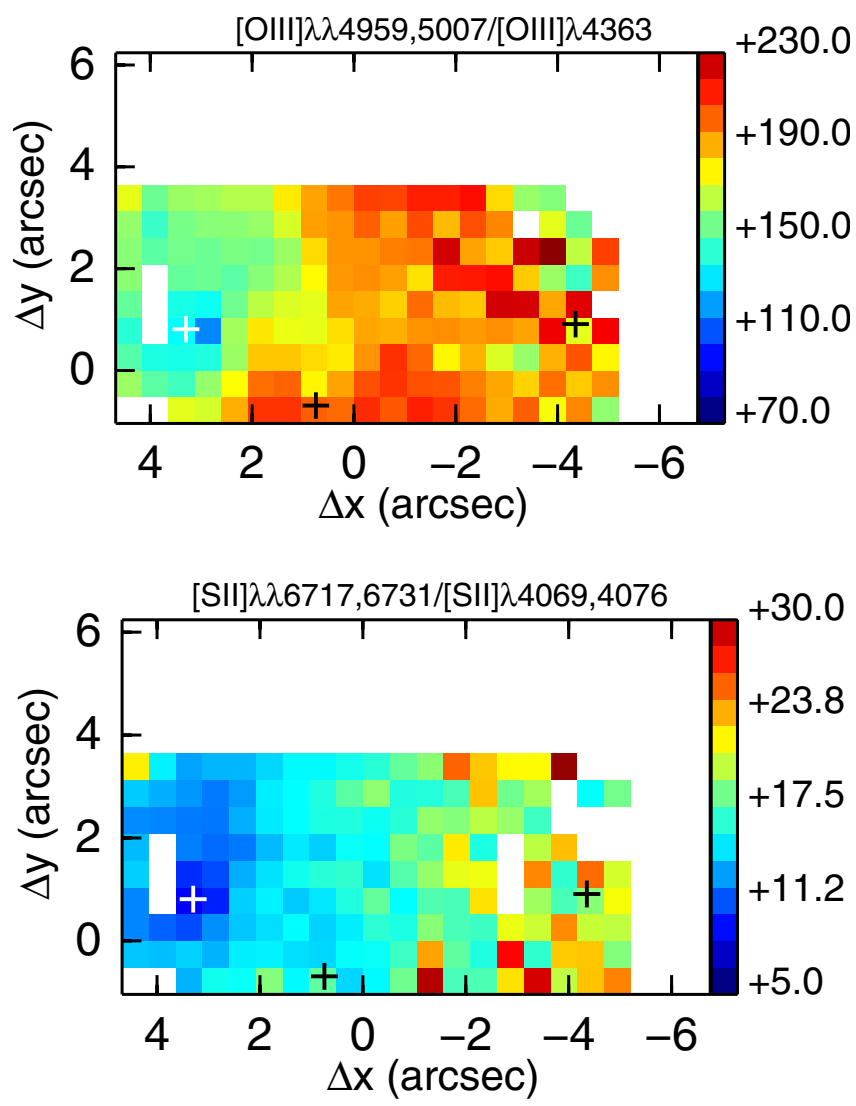

Fig. 5. Maps for the line ratios sensitive to $T_{\mathrm{e}}$ tracing the high (upper) and the low (lower) ionization regime. The position of the three main peaks of continuum emission are shown as crosses for reference.

from the one using the $\left[\mathrm{S}_{\mathrm{II}}\right]$ lines. We will explore in Sect. 4 how this is related to the ionization structure of the galaxy.

\subsection{Mapping tracers of the local ionization degree}

The relation between the $[\mathrm{N}$ II $] \lambda 6584 / \mathrm{H} \alpha$ and $\left[\mathrm{S}_{\mathrm{II}}\right] \lambda \lambda 6717$, $6731 / \mathrm{H} \alpha$ line ratios in galaxies is not monotonic, even though the $\mathrm{S}^{+}$and $\mathrm{N}^{+}$ionization potentials and critical densities are in a similar range. Instead, different ionization mechanisms (shocks, star formation, AGNs, e.g. Monreal-Ibero et al. 2006, 2010a) and/or physical and chemical conditions of the gas (metallicity, relative abundances and degree of ionization) trace different loci in the $[\mathrm{N} I I] \lambda 6584 / \mathrm{H} \alpha$ vs. $[\mathrm{S}$ II] $\lambda \lambda 6717,6731 / \mathrm{H} \alpha$ diagram. In Paper I, we attributed the different structure in the $\left[\mathrm{N}_{\text {II }}\right] \lambda 6584 / \mathrm{H} \alpha$ and $\left[\mathrm{S}_{\mathrm{II}}\right] \lambda \lambda 6717,6731 / \mathrm{H} \alpha$ maps to an excess in nitrogen. This was supported by evidence of star-formation as the main ionization mechanism and previous estimations of the ionization parameter at specific locations based on long-slit measurements (Kobulnicky et al. 1997). However, certain combinations of specific ionization structures and metallicity gradients could reproduce a similar locus in the $\left[\mathrm{N}_{\mathrm{II}}\right] \lambda 6584 / \mathrm{H} \alpha$ vs. [S II] $\lambda \lambda 6717,6731 / \mathrm{H} \alpha$ diagram. This possibility can be ruled out with the present data. Here, we present the ionization structure in NGC 5253 as traced by different line ratios, while the next section will contain maps for the abundances of different heavy elements (oxygen, nitrogen, neon and argon).

The degree of ionization can be traced by means of ratios of lines of the same element tracing two different ionization states. The upper panel of Fig. 6 contains a map for one of these ratios: [O III] $] \lambda 44959,5007 /\left[\mathrm{O}_{\text {III }}\right] \lambda \lambda 3726,3729$. Note that in order to 
A. Monreal-Ibero et al.: Physical and chemical conditions of the ionized gas in NGC 5253
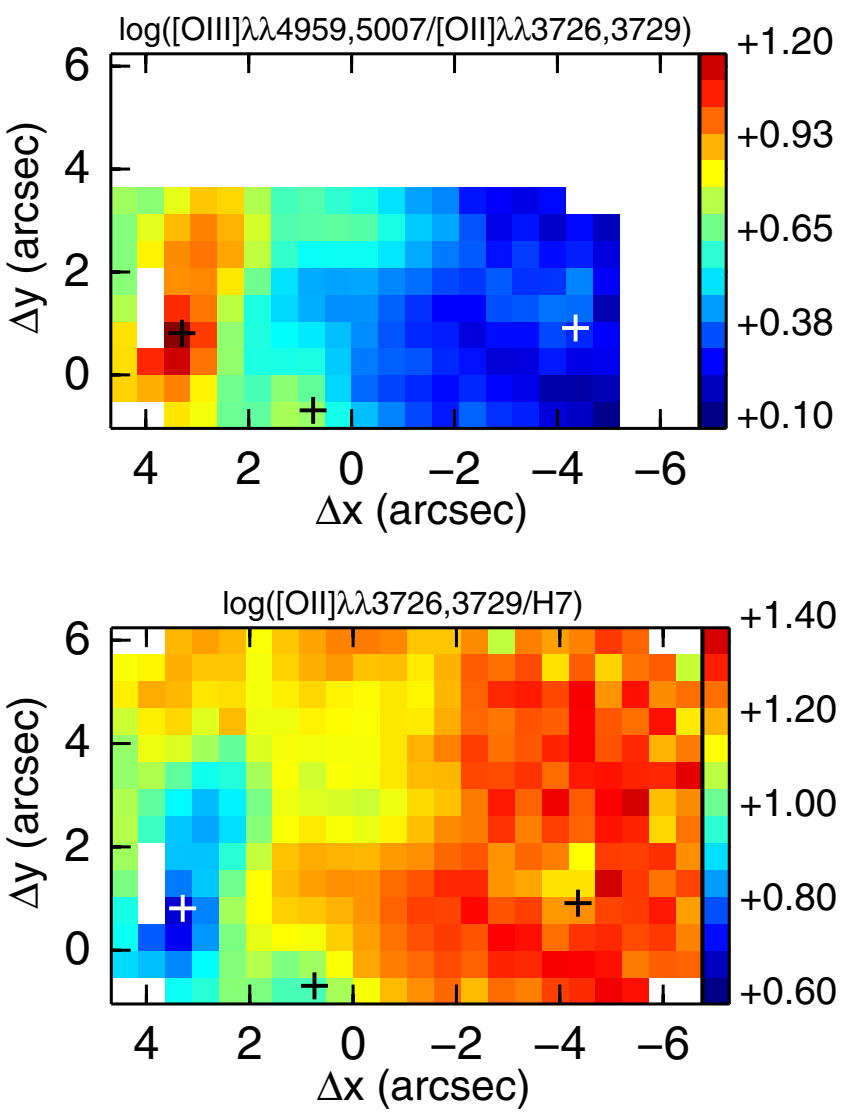

Fig. 6. Maps for two tracers of the ionization degree. Top: $\left[\mathrm{O}_{\mathrm{III}}\right] /\left[\mathrm{O}_{\mathrm{II}}\right]$. Bottom: $[\mathrm{O}$ II $] / \mathrm{H} 7$. The position of the three main peaks of continuum emission are shown as crosses for reference.

minimize uncertainties associated to aperture matching, absolute flux calibration and extinction, the $\left[\mathrm{O}_{\mathrm{III}}\right]$ and $\left[\mathrm{O}_{\mathrm{II}}\right]$ lines were measured relative to $\mathrm{H} \beta$ and $\mathrm{H} 7$, respectively. Then, we assumed the theoretical Balmer line intensities obtained from Storey \& Hummer (1995) for Case B, $T_{\mathrm{e}}=10^{4} \mathrm{~K}$ and $n_{\mathrm{e}}=100 \mathrm{~cm}^{3}$. The map shows that the ionization structure reproduces the morphology observed for the ionized gas, thus: i) line ratios tracing the highest ionization degree are associated to knot $\sharp 1$; ii) relatively high ionization is found in the main GH IIR; iii) a secondary peak of high ionization is found around knot $\sharp 2$; iv) low ionization degree is found in the rest of the fov, where the diffuse component of the ionized gas becomes more relevant.

Also, if the metallicity is known, line ratios like [O II $] \lambda \lambda 3726,3729 / \mathrm{H} 7$ or $\left[\mathrm{S}_{\text {II }}\right] \lambda \lambda 6717,6731 / \mathrm{H} \alpha$ can also be used to trace the ionization degree. The map [O II] $\lambda \lambda 3726,3729 / \mathrm{H} 7$ is presented in the lower panel of Fig. 6 while that of [S II] $\lambda \lambda 6717,6731 / \mathrm{H} \alpha$ was included in Paper I. The similar structure in all the three maps suggests a lack of metallicity gradient in the galaxy as will be shown in the next section.

In order to explore whether these three tracers predict consistent ionization degree for a given position, we show in Fig. 7, the relation between the different ratios for each individual spaxel, using [O III] $] \lambda 44959,5007 /\left[\mathrm{O}_{\text {II }}\right] \lambda \lambda 3726,3729$ as reference. Also, we overplotted the locus of equal estimated ionization parameter according to the relations proposed by Díaz et al. (2000) and assuming $Z=0.3 Z_{\odot}$ with a black line. For a given location, [O III] $] \lambda \lambda 4959,5007 /[\mathrm{O}$ II $] \lambda \lambda 3726,3729$ and $\left[\mathrm{O}_{\mathrm{II}}\right] \lambda \lambda 3726,2729 / \mathrm{H} \beta$ predict a similar degree of ionization while the predictions of $\left[\mathrm{S}_{\mathrm{II}}\right] \lambda \lambda 6717,6731 / \mathrm{H} \alpha$ would correspond to smaller ionization parameters. A similar result was
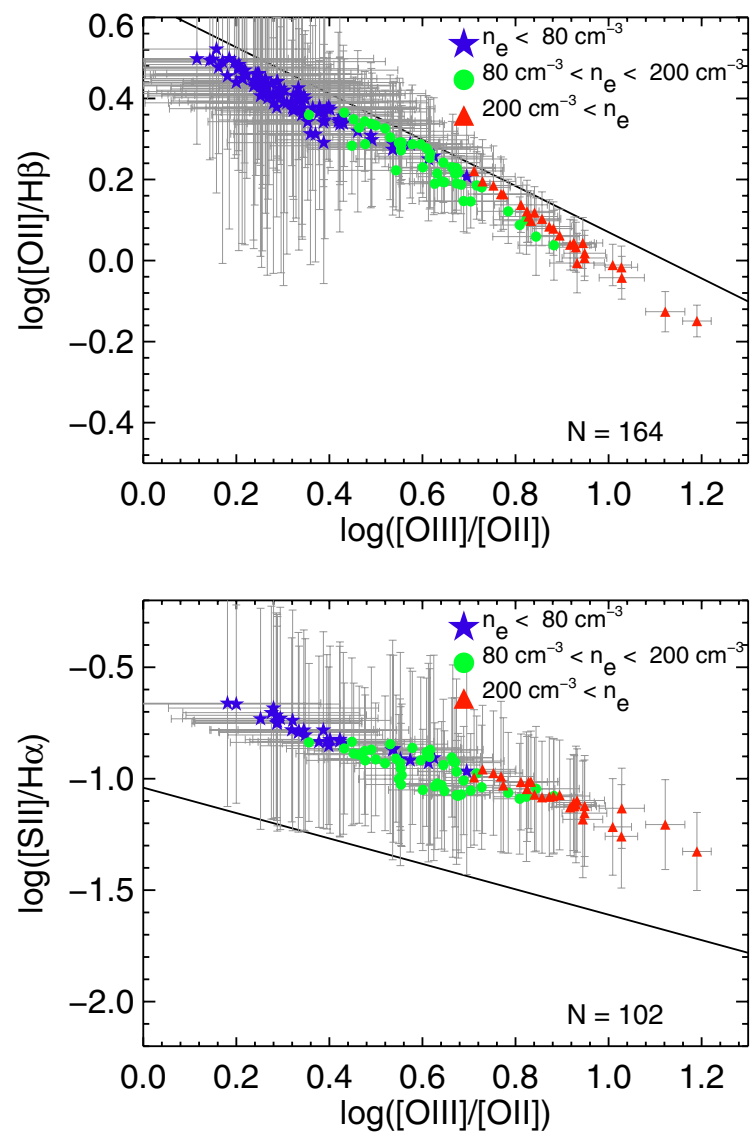

Fig. 7. Diagrams involving two ratios sensitive to the ionization degree. Top: [O III] $\lambda \lambda 4959,5007 /\left[\mathrm{O}_{\text {II }}\right] \lambda \lambda 3726,3729$ vs. [O II] $] \lambda 3726,3729 / \mathrm{H} \beta$. Bottom: [S II] $\lambda \lambda 6717,6731 / \mathrm{H} \alpha$ vs. [O III] $\lambda \lambda 4959,5007 /$ [O II] $\lambda \lambda 3726$, $3729)$. The locus of equal estimated ionization parameter according to the relations proposed by Díaz et al. (2000) and assuming $Z=0.3 Z_{\odot}$ is indicated with a black line. Only those spaxels with estimated uncertainties for the involved ratios smaller than 0.5 dex have been taken into account. Spaxels were grouped in three bins according their $n_{\mathrm{e}}\left(\left[\mathrm{O}_{\mathrm{II}}\right]\right)$ as indicated in the upper right-hand corner of the individual diagrams. The total number of considered data points is indicated in the lower right-hand corner.

found in a detailed analysis of NGC 588, a GH IIR in M 33 (Monreal-Ibero et al. 2011a). Both, the spatial variations of the different line ratios (Fig. 6) and the observed excess in the [S II] $\lambda \lambda 6717,6731 / \mathrm{H} \alpha$ ratio when compared with photoionization models (Fig. 7) can be jointly explained as a 3D view of the ionization structure of the galaxy. Specifically, for a given spaxel (i.e. a given line of sight), the lower ionization species (e.g. $\mathrm{S}^{+}$), delineate the more extended diffuse component while $\mathrm{O}^{++}$will be confined to different high ionization zones closer to the ionizing sources. Alternatively, the difference between the ratios found here and those predicted by the Díaz et al. models can be attributed to differences between the relative $\mathrm{S} / \mathrm{O}$ abundances in NGC $5253(\log (\mathrm{S} / \mathrm{O}) \sim-1.47$, Kobulnicky et al. 1997; Sidoli $2010)$ and those assumed in the models $(\log (\mathrm{S} / \mathrm{O})=-1.71$, Grevesse \& Anders 1989). A detailed modelling of the ionization structure of the galaxy - out of the scope of this work could help to disentangle these two possibilities.

\subsection{Mapping abundances of heavy elements}

Following the same methodology as in Sect. 3.1.2, we derived maps for the abundances of oxygen, nitrogen, neon and argon. 

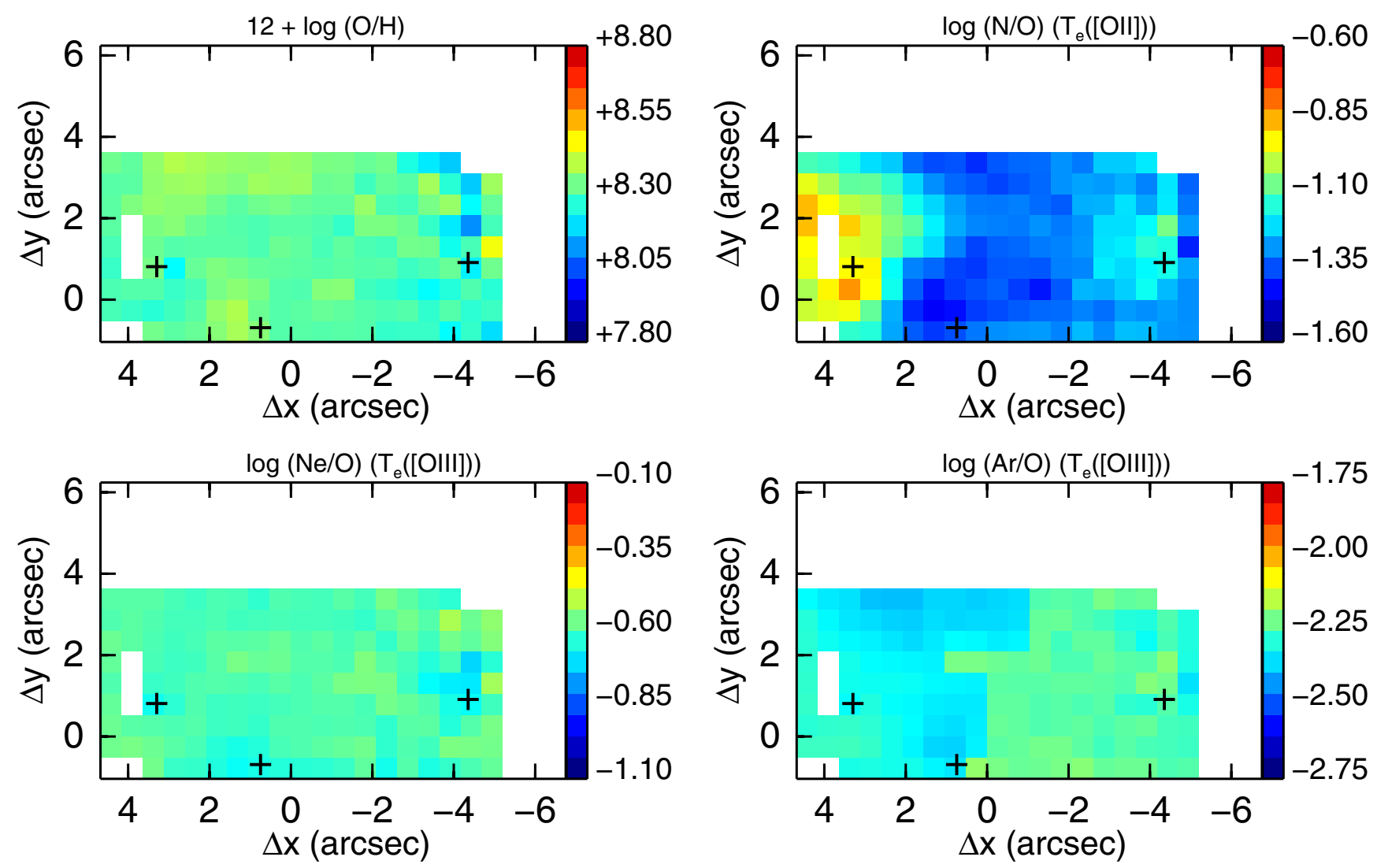

Fig. 8. Metallicity and relative abundances maps. From top to bottom and left to right: oxygen abundance, and nitrogen, neon and argon relative abundances with respect to oxygen. In order to make easier the comparison between the different maps, all of them cover a range of 1 dex.

These are presented in Fig. 8 and show, as expected, a homogeneous distribution within the uncertainties in all the elements but nitrogen. Note that the spaxel-to-spaxel variations in these maps are not dominated by the quality of the data (e.g. $\mathrm{S} / \mathrm{N}$ ratio), but are inherent to the adopted $T_{\mathrm{e}}$ (and to a much lesser extent $n_{\mathrm{e}}$ ) as well as the utilized methodology. The most obvious example would be the map showing the distribution of the argon abundance, where a different methodology was adopted to estimate the ICF depending on whether the [Ar IV] $\lambda 4740$ emission line was or was not detected (see Fig. 6 in Paper I, to locate those spaxels with detection). Nevertheless, abundances derived in these two groups differ only by $\sim 0.1$ dex, well within the expected uncertainties for this element. Also, $12+\log (\mathrm{O} / \mathrm{H})$ marginally anti-correlates with $T_{\mathrm{e}}([\mathrm{O} \mathrm{III}])$ in the spaxels with lowest surface brightness. The mean $( \pm$ standard deviation) metallicity in our fov is $12+\log (\mathrm{O} / \mathrm{H})=8.26$ $( \pm 0.04)$ while the relative abundances $\log (\mathrm{Ne} / \mathrm{O})$ and $\log (\mathrm{Ar} / \mathrm{O})$ are $-0.64( \pm 0.03)$ and $-2.32( \pm 0.05)$. Supporting this homogeneity, in all three cases - oxygen, neon and argon - the distribution of the values measured over the fov can be well reproduced by a Gaussian with $\sigma \sim 0.03-0.07$ dex and all the values measured in the individual spaxels are consistent with the mean value at the $3 \sigma$ level.

The abundance in nitrogen differs from this pattern. As reported in Paper I, there is a roughly elliptical area of $\sim 80 \mathrm{pc} \times$ $35 \mathrm{pc}$, associated to the main $\mathrm{H}$ in region and centered on the two massive SSCs, that presents an enhancement of N/O. More interestingly, a putative secondary excess in nitrogen not reported so far appears in the vicinity of (but not centered on) knot $\sharp 3$, at the area of our fov with the lowest surface brightness.

The existence of a second area with slight N/O enhancement is supported by the histogram of the values measured along the FLAMES fov (not shown). Contrary to the case for $\mathrm{O}, \mathrm{Ne}$ and $\mathrm{Ar}$, three Gaussians centered at $\log (\mathrm{N} / \mathrm{O})=-1.32,-1.17$ and -0.95 and with widths $\sigma=0.05,0.07,0.03$ are needed to reproduce the full distribution. Most of the spaxels are associated to the first Gaussian and trace the typical N/O abundance for NGC 5253. The second largest group, with $\log (\mathrm{N} / \mathrm{O}) \sim-0.95$, are associated to the area with $\mathrm{N}$-enhancement already reported for the main GH IIR. Using the criterion that spaxels belonging to two Gaussian trace different abundances if their centers are separated by more than $3 \sigma$, we can conclude that the third group of spaxels, which are associated to the area in the vicinity of knot $\sharp 3$, traces a zone with slightly larger, but clearly distinct, N/O value than the typical one for the galaxy. Given that this area is located at the spaxels with the lowest surface brightness, and to reject the possibility of any systematic effect due to a poor $\mathrm{S} / \mathrm{N}$ ratio in faint lines like [O $\mathrm{III}] \lambda 4363$, we extracted a spectrum by summing up the flux in a rectangular area of $5 \times 6$ spaxels at this location. The measured relative $\mathrm{N}$-abundance, $\log (\mathrm{N} / \mathrm{O})=-1.15$, is in agreement with the result found on a spaxel-by-spaxel basis.

\section{Discussion}

\subsection{Electron density structure}

As we discussed in Sect. 3.3, for a given spaxel, different ions are associated to different layers along the line of sight. We have presented maps for tracers of electron density based on [S II], [Fe III $]$ and [O II] emission line ratios (Paper I, and Sect. 3.2). The corresponding density maps were derived using the same methodology as in Sect. 3.1.1 and appear in Fig. 9. Values consistent with being below the low-density limit are found in most 

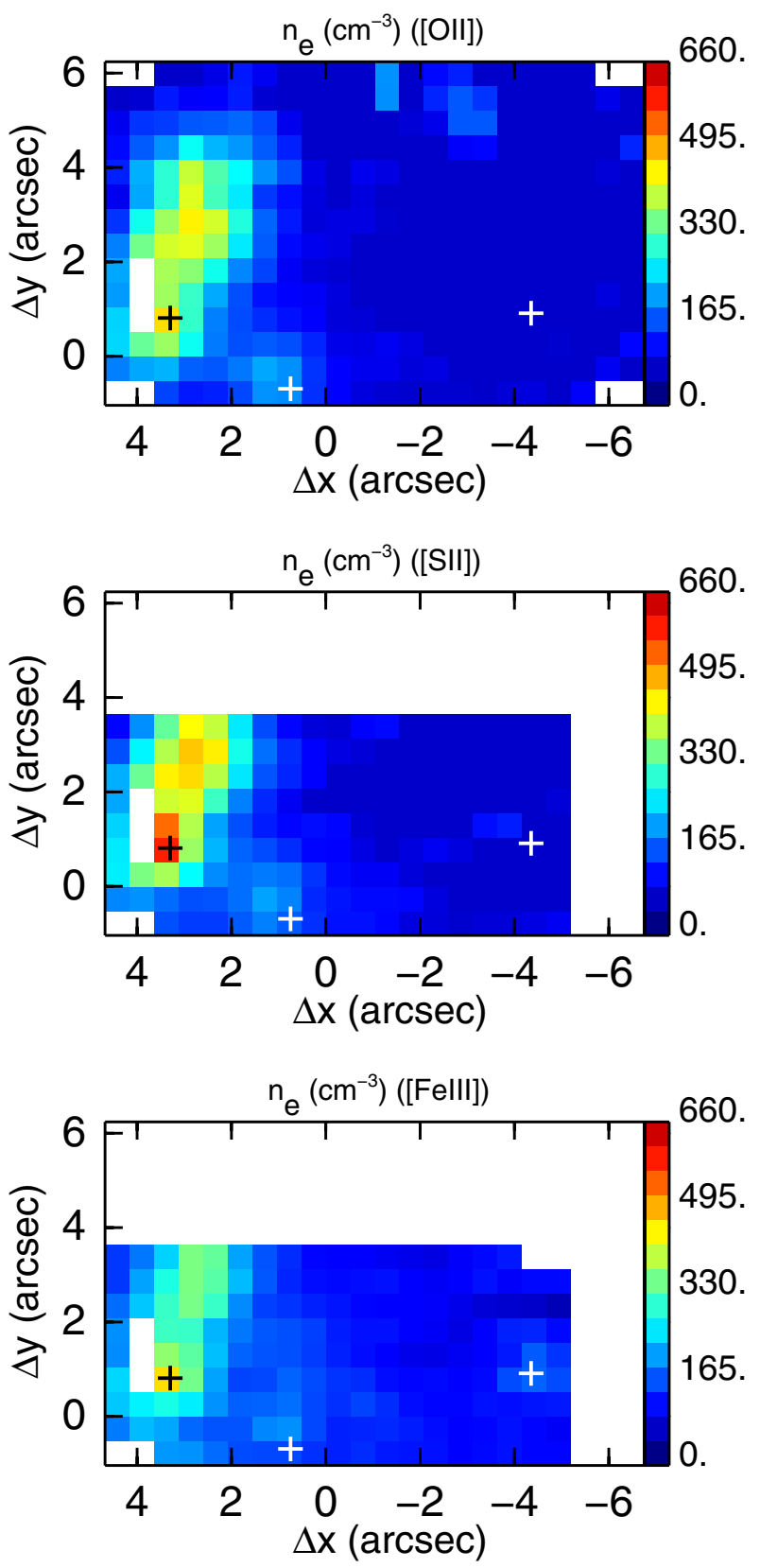

Fig. 9. Electron density maps from different tracers. From top to bottom: [O II], [S II], and [Fe III]. The position of the three main peaks of continuum emission are shown as crosses for reference.

of the fov, independently of the utilized tracer, while knot $\sharp 2$ presents values somewhat larger $\left(\sim 190 \mathrm{~cm}^{-3}\right)$. The richest density structure is found in the main GH IIR. Here, all three tracers depict an $n_{\mathrm{e}}$ structure with two peaks: the first one centered at knot $\sharp 1$ while the second one at $\sim 22^{\prime \prime} 6(\sim 50 \mathrm{pc})$ towards the northwest. However the values of the peaks vary depending on the utilized tracer. The largest densities in Fig. 9 are traced by [S II], while [O II] and [Fe III] predict densities about $10-25 \%$ smaller. Given their ionization potentials $(23.3,35.1,30.7 \mathrm{eV}$ for [S $\mathrm{II}],\left[\mathrm{O}_{\mathrm{II}}\right]$ and $[\mathrm{Fe} \mathrm{III}]$, respectively), in the absence of extinction, $n_{\mathrm{e}}([\mathrm{O} \mathrm{II}])$ - for example - traces the density in a layer closer to the ionizing source than $n_{\mathrm{e}}\left(\left[\mathrm{S}_{\mathrm{II}}\right]\right)$ (see e.g. Garnett 1992). This can be reversed in conditions of heavy extinction. Even if the intrinsic structure would be the same, lines involved in the determination of $n_{\mathrm{e}}([\mathrm{O} \mathrm{II}])$ are bluer (and therefore more sensitive to extinction) than those associated to $n_{\mathrm{e}}([\mathrm{S} \mathrm{II}])$ which therefore can
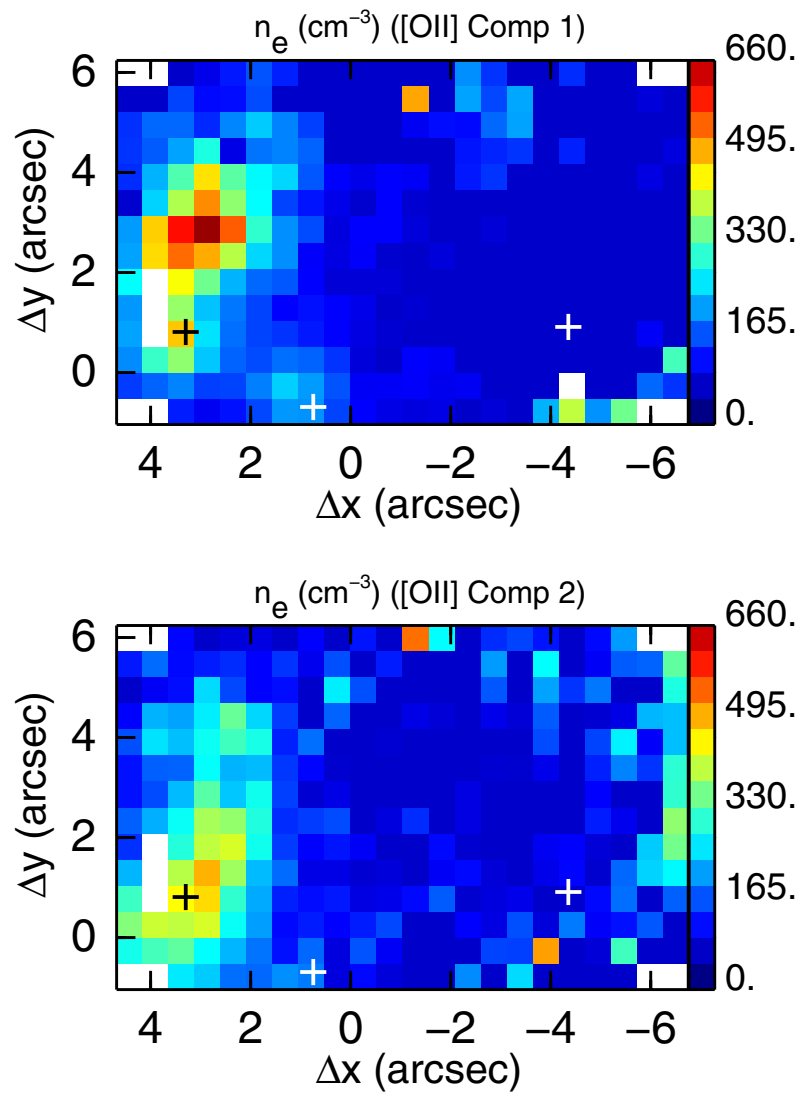

Fig. 10. Electron density maps for the different components using the results for [O II]. Top: narrow component; bottom: broad component. The position of the three main peaks of continuum emission are shown as crosses for reference.

probe deeper in the nebula. Since this GH IIR suffers from relatively high extinction (see e.g. Fig. 3. in Paper I) the differences found between the three density maps are consistent with an onion-like structure where inner layers are denser than the outer ones. This is supported by the relative densities found between the two peaks. Also it is consistent with the fact that the [Fe III] ratio does not trace very high densities as the [Ar IV] ratio did (Paper I). With an ionization potential of $59.8 \mathrm{eV}$, [Ar IV] would sample the densest innermost layer of this onion-like structure.

The one Gaussian fitting approach utilized up to now is useful to have a picture of the overall density structure in the galaxy. However, it is known that emission lines in NGC 5253 present complex profiles with asymmetries tracing different kinematics components. For the GH IIR, we tentatively measured the electron density for what we called the "broad" and "narrow" components on a spaxel-by-spaxel basis in Paper I using the [S II] lines. Results were affected by large uncertainties, mainly due to the deblending procedure but also to the $\mathrm{S} / \mathrm{N}$ ratio of the spectra. They suggested large and uniform densities for the "broad" component $\left(n_{\mathrm{e}} \sim 470 \mathrm{~cm}^{-3}\right)$, while the "narrow" component presented a decrease of density from the northwest to the southeast. The stronger [O $\mathrm{O}_{\mathrm{II}}$ ] lines offer the possibility of discussing this difference with much reduced uncertainties. Electron density maps from our multi-component analysis are presented in Fig. 10. The structure depicted in the map for broad component (bottom panel), with larger densities $\left(\sim 500 \mathrm{~cm}^{-3}\right)$ close to knot $\sharp 1$ and decreasing outwards, is consistent with the general picture sketched above (i.e. larger densities closer to the ionizing source). The narrow component (upper panel) present a 

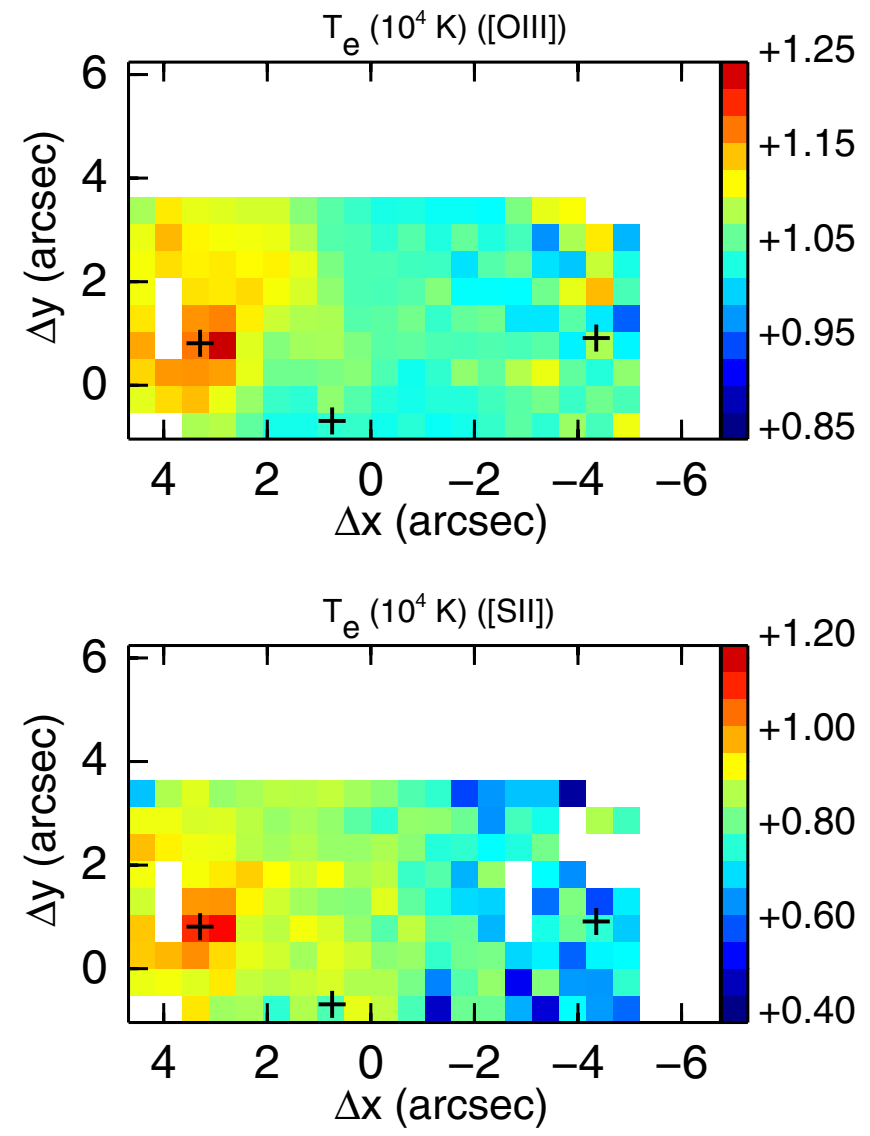

Fig. 11. Electron temperature maps from different tracers. Top: [O III]; bottom: $[\mathrm{S}$ II]. The position of the three main peaks of continuum emission are shown as crosses for reference.

different structure, with the highest density $\left(\$ 600 \mathrm{~cm}^{-3}\right)$ at the secondary peak of the GH IIR at $\sim 2$ '. 6 from knot $\sharp 1$. This can be understood within the proposed scenario in Paper I (see their Fig. 21, where different elements associated with the area of the GH IIR are shown). There, this narrow component was associated to a shell of previously existing quiescent gas that has been reached by the ionization front. Densities in the shell would be high, if this pre-existing gas had been piled-up by the outflow associated to the broad component.

\subsection{Electron temperature structure}

As it happened with $n_{\mathrm{e}}$, a comparison of $T_{\mathrm{e}}$ maps derived from lines associated to different ions is useful to asses the $T_{\mathrm{e}}$ structure in 3D of NGC 5253. Derived maps for $T_{\mathrm{e}}([\mathrm{O} \mathrm{III}])$ and $T_{\mathrm{e}}([\mathrm{S} \mathrm{II}])$ are presented in Fig. 11. At present, maps of $T_{\mathrm{e}}$ based on any tracer are still scarce and most of the time focused on Galactic objects (e.g. Núñez-Díaz et al. 2012; Tsamis et al. 2008). Indeed, to our knowledge this is the first time that a map for $T_{\mathrm{e}}([\mathrm{S} \mathrm{II}])$ is provided and one of the few existing examples of $T_{\mathrm{e}}([\mathrm{O} \mathrm{III}])$ maps in extragalactic astronomy. As we pointed out in Sect. 3.2, the overall structure is the same for $T_{\mathrm{e}}([\mathrm{O} \mathrm{III}])$ and $T_{\mathrm{e}}\left(\left[\mathrm{S}_{\mathrm{II}}\right]\right)$ maps. However, $T_{\mathrm{e}}\left(\left[\mathrm{S}_{\mathrm{II}}\right]\right)$ is smaller than $T_{\mathrm{e}}\left(\left[\mathrm{O}_{\mathrm{III}}\right]\right)$, with typical $T_{\mathrm{e}}([\mathrm{S} \mathrm{II}]) / T_{\mathrm{e}}([\mathrm{O}$ III $])$ ranging from $\sim 0.8$ at the $\mathrm{GH}$ IIR to $\sim 0.6$ in the areas of low surface brightness.

This relation between both temperatures is shown on a spaxel-by-spaxel basis in Fig. 12. Only those spaxels where the estimated uncertainties for both temperatures are smaller than $40 \%$ were considered. Both, the different values for $T_{\mathrm{e}}([\mathrm{O} \mathrm{III}])$

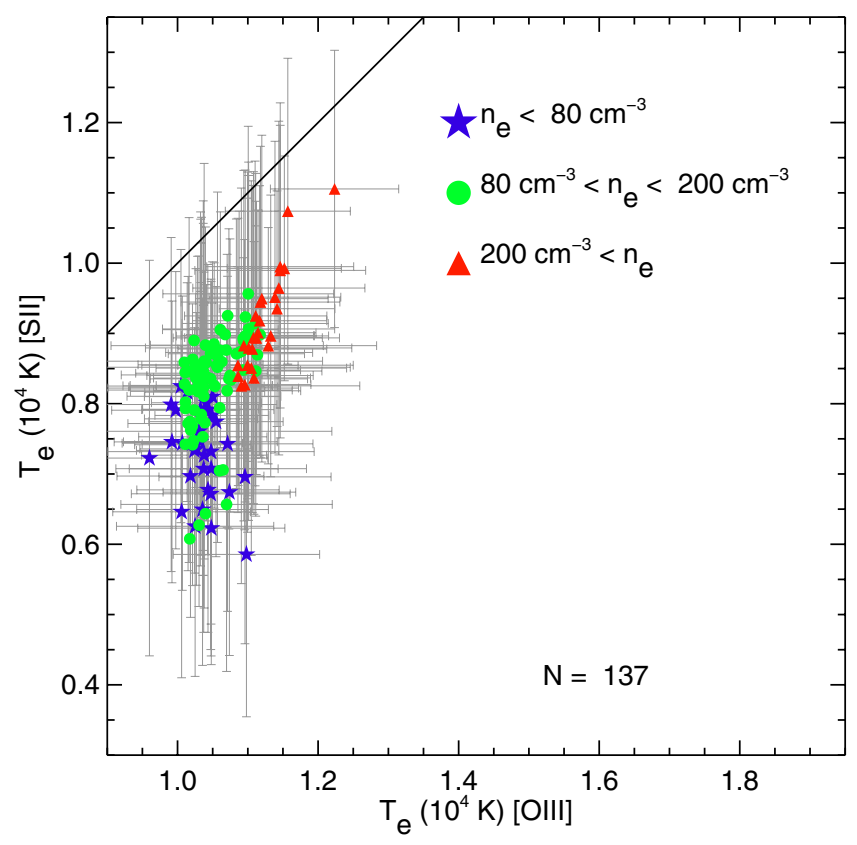

Fig. 12. Comparison between the predicted $T_{\mathrm{e}}\left(\left[\mathrm{O}_{\mathrm{III}}\right]\right)$ and $T_{\mathrm{e}}\left(\left[\mathrm{S}_{\mathrm{II}}\right]\right)$. Spaxels were grouped in the same three bins in $n_{\mathrm{e}}([\mathrm{O} \mathrm{II}])$ as in Fig. 7 as it is indicated in the upper right-hand corner of the diagram.

and $T_{\mathrm{e}}([\mathrm{S} \mathrm{II}])$ and the larger differences at low surface brightness, can be understood within the frame of a change in the relative contribution along the line of sight of the warmer gas associated to the GH IIR and a colder and more diffuse gas component that extends further away. The 2D information on the plane of the sky (i.e. the maps in Fig. 11) fits also in a satisfactory manner with this interpretation.

Summarizing, both the available information on the plane of the sky (Fig. 11) and along the line of sight (Fig. 12) are consistent with a $T_{\mathrm{e}}$ structure in $3 \mathrm{D}$ with higher temperatures close to the ionizing source surrounded by a more diffuse component of ionized gas at lower temperatures.

\subsection{Validity of strong line methods: $N / O$ relative abundance}

Ideally, metallicity and relative abundances for the ionized gas in galaxies should be derived in a direct manner. However, this requires the determination of $T_{\mathrm{e}}$, which depends on the detection of faint lines like [O III] $\lambda 4363$. In extragalactic astronomy, most of the time this is not feasible. Instead, certain combinations of strong emission lines with more or less well established empirical and/or theoretical calibrations should be used (e.g. Pérez-Montero \& Contini 2009; Kewley \& Ellison 2008). A comparison between both methodologies on a spaxel-by-spaxel basis would be useful to test the reliability of the strong linebased methods and identify the cause of possible biases. For example, Monreal-Ibero et al. (2011a) and Relaño et al. (2010) found that metallicity tracers are modulated by the ionization structure. The best-known metallicity tracer would probably be R23 (Pagel et al. 1979). Unfortunately, with a $12+\log (\mathrm{O} / \mathrm{H}) \sim$ 8.28 , NGC 5253 falls at the turn-over of the metallicity-R23 relation and therefore, this tracer is not appropriate for metallicity determinations in this galaxy. Alternatively, two widely used tracers are $03 N 2=\log (([\mathrm{O}$ III $] \lambda 5007 / \mathrm{H} \beta) /([\mathrm{N} \mathrm{II}] \lambda 6584 / \mathrm{H} \alpha))$ (Alloin et al. 1979) and $\mathrm{N} 2=[\mathrm{N}$ II $] \lambda 6584 / \mathrm{H} \alpha$ (Denicoló et al. 2002). However, both involve [ $\left.\mathrm{N}_{\mathrm{II}}\right]$ emission lines, and are therefore, affected by the variation of relative $\mathrm{N} / \mathrm{O}$ abundance 

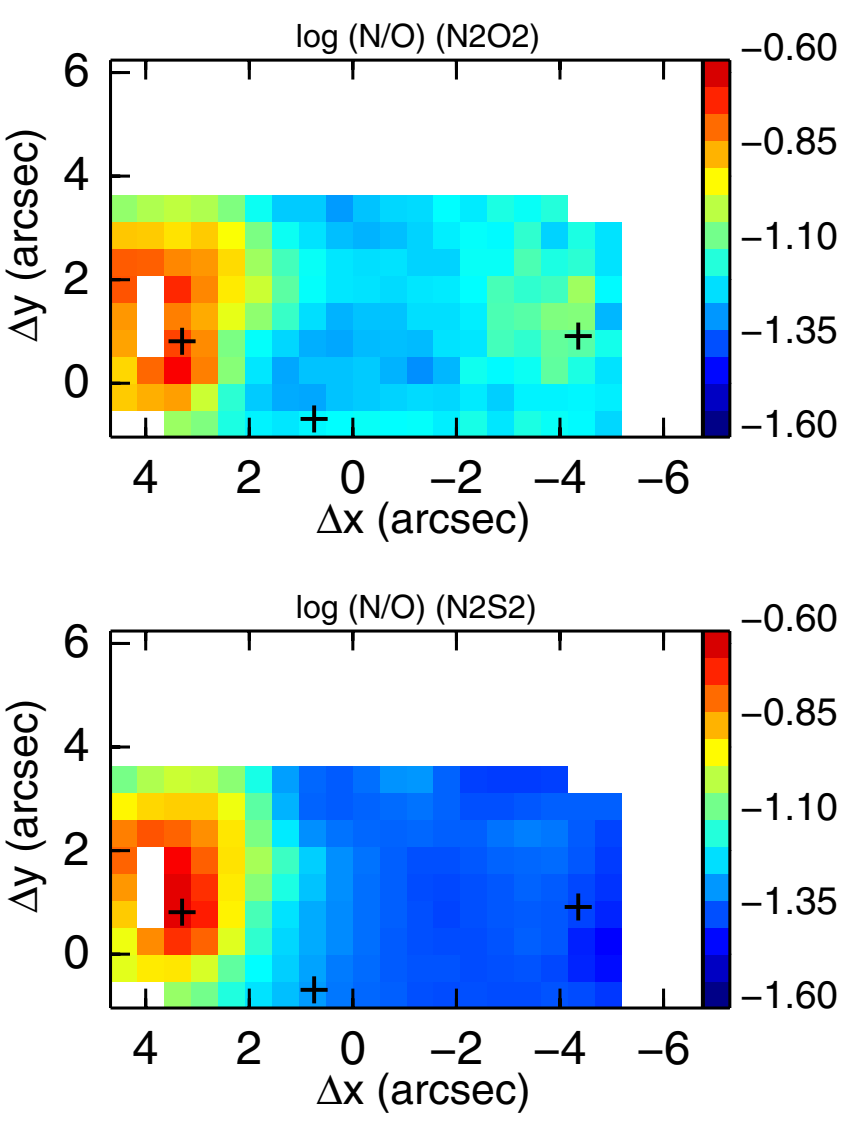

Fig. 13. Predicted N/O maps using the $\mathrm{N} 2 \mathrm{O} 2$ (top) and $\mathrm{N} 2 \mathrm{~S} 2$ (bottom) tracers. Note that for an easier comparison with the predictions of the direct methods, maps are displayed using a scale covering the same range as the one presented in Fig. 8.

across the galaxy. Thus, NGC 5253 is not an adequate example to test the reliability of metallicity determinations based on strong optical lines. Instead, since this galaxy presents a range of $\mathrm{N} / \mathrm{O}$ abundances, a different test for the corresponding strong line based tracers would be of high interest. This will be discussed in this section. As a baseline, we converted our line ratio maps into relative abundances using the expressions provided by Pérez-Montero \& Contini (2009), that relate $\mathrm{N} / \mathrm{O}$ with $\mathrm{N} 2 \mathrm{O} 2=\log \left(\left[\mathrm{N}_{\text {II }}\right] \lambda 6584 /\left[\mathrm{O}_{\text {II }}\right] \lambda \lambda 3726,3729\right)$ and $\mathrm{N} 2 \mathrm{~S} 2=\log \left(\left[\mathrm{N}_{\mathrm{II}}\right] \lambda 6584 /\left[\mathrm{S}_{\mathrm{II}}\right] \lambda \lambda 6717,6731\right)$ as defined by Kewley \& Dopita (2002) and Sabbadin et al. (1977).

Maps for the estimated relative N/O abundances are presented in Fig. 13. They show that both tracers, N2O2 and N2S2, detect the main area with $\mathrm{N}$-enhancement. Interestingly enough, only $\mathrm{N} 2 \mathrm{O} 2$ is sensitive to the newly discovered area, associated to knot $\sharp 3$.

However, both tracers fail to predict the correct N/O abundance. This is not unexpected since the utilized relations are valid to interpret global tendencies in a statistically significant sample of galaxies, while N/O abundances for an individual object can depart about \pm 0.3 and \pm 0.5 dex from this relation for the $\mathrm{N} 2 \mathrm{O} 2$ and N2S2 tracers respectively (see e.g. Figs. 10 and 11 in Pérez-Montero \& Contini 2009).

Figure 14 shows a comparison of the residuals between the strong line methods and the direct one. Only those spaxels with an estimated uncertainty lower than 0.25 dex in both residuals were included in the comparison. The good correlation between the residuals is consistent with both methods being affected by the same factors. Also, there is an increase of the

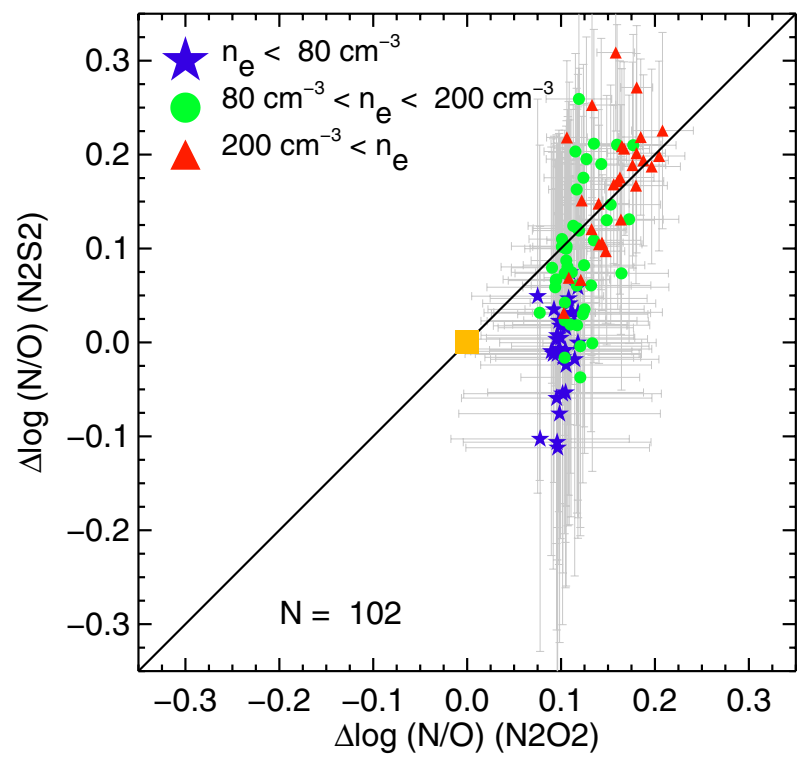

Fig. 14. Comparison between the residuals for the determination of N/O based on the two strong line methods under consideration with respect to the direct one. The locus of equal residuals is indicated with a black line. An orange square marks the position where the three methods agree. Data were divided in three bins of $n_{\mathrm{e}}$ as in Figs. 7 and 12.

residual with $n_{\mathrm{e}}$. This also implies an increase with the other physical parameters since a comparison of the different maps presented throughout the paper shows that in general, larger $n_{\mathrm{e}}$ corresponds to brighter areas and with larger $T_{\mathrm{e}}$ and ionization strength. Given the spatial coincidence of these variations, disentangling the relative role of a given physical quantity as the cause of the variations in the residuals is not straightforward for this galaxy and will not be addressed here. Mean $( \pm$ standard deviation) for the residuals are $\sim 0.11( \pm 0.03)$ and $\sim 0.02( \pm 0.12)$ for $\mathrm{N} 2 \mathrm{O} 2$ and $\mathrm{N} 2 \mathrm{~S} 2$, respectively. Therefore, a method based on $\mathrm{N} 2 \mathrm{O} 2$ is $\sim 4$ times less sensitive to any variation of the physi$\mathrm{cal} /$ chemical properties than the one based on N2S2.

In summary, our comparison of the N/O abundances derived using strong line methods with respect to direct measurements in the search for chemical inhomogeneities within a galaxy, supports the use of that based on N2O2 over that based on N2S2. The first method is sensitive to a wider range of N/O abundances and is more stable against variations of physical conditions within the area of interest.

\subsection{On the relationship between extra $N$ and WR stars}

There are several works in the literature using long-slit observations that suggest a connection between nitrogen overabundance and the presence of WR stars based on the simultaneous detection of WR features and a higher than the expected N/O in a given object (e.g. Walsh \& Roy 1987; Thuan et al. 1996; Guseva et al. 2000; Pustilnik et al. 2004; Izotov et al. 2006b; Hägele et al. 2006; Pérez-Montero et al. 2010; López-Sánchez \& Esteban 2010). Also, local associations between WR emission and N-enhancements have been found by means of IFS based observations (e.g. James et al. 2009) supporting this connection. However, the general picture is far from this simple one-to-one association. For example, Pérez-Montero et al. (2011) studied a sample of BCD galaxies that are N-overabundant over large areas supporting the idea of enrichment caused by accretion of less chemically processed gas (see also Kehrig et al. 2008). A similar 
result was found by Amorín et al. (2012) for a set of BCD galaxies at higher redshift. On top of that, resolution effects also play a important role. Already at distances of $\sim 25-40 \mathrm{Mpc}$ the whole area covered here would have a projected size of the order of the resolution of typical ground based observations $\left(\sim 1^{\prime \prime} .0\right)$.

We reported in Paper I areas with both extra- $\mathrm{N}$ and WR emission in NGC 5253, as for example, our knot $\sharp 1$ (widely discussed in the literature, e.g. Walsh \& Roy 1989; Kobulnicky et al. 1997; Schaerer et al. 1999; López-Sánchez et al. 2007) together with areas with WR emission but without any chemical anomaly (e.g. our knot $\sharp 2$ ). Moreover, we report in Sect. 3.4 a new area presenting an overabundance of nitrogen without any WR emission associated to it. This variety of options (i.e. WR emission and enhanced $\mathrm{N}$, no WR emission and enhanced $\mathrm{N}$, WR emission and no enhanced $\mathrm{N}$, no WR emission and no enhanced $\mathrm{N}$ - i.e. the normal case) seems a more natural situation than a one-toone association since the chemical enrichment of the warm ionized medium due to the material expelled by the massive stars is a complex process. In a simplified manner, this can be divided into three basic steps.

Firstly, stars must bring their processed material to the surface. Recent stellar evolution models show how the inclusion of rotation favors the appearance of processed material at stages as early as the main sequence (i.e. Maeder \& Meynet 2000; Meynet \& Maeder 2005; Maeder \& Meynet 2010). However they are particularly powerful at the WR, or even Luminous Blue Variable, phases (see Crowther 2007, and references therein). Also, observational evidence of N-enhancement in WR ring nebulae has been reported (Fernández-Martín et al. 2012).

Secondly, this material should be expelled via stellar winds, first, and supernova explosions, afterwards. In the case of star clusters, where stars with a variety of initial masses have a variety of evolutionary paths, the yield of the different elements varies in a non-trivial manner with time (e.g. Mollá \& Terlevich 2012). An extra caveat arises for clusters with stellar masses of $\lesssim 10^{4} M_{\odot}$ since in this regime the Initial Mass Function is not properly sampled and stochastic effects are important (e.g. Villaverde et al. 2010).

Finally, this new material must be disseminated and then diluted in the warm ionized medium which, in due time, would reach a new chemical homogeneity. This is an even less trivial step since on top of the specific characteristics of a given star cluster, factors like the existence and characteristics of neighbouring clusters, presence of cloudlets of gas, degree of the inhomogeneities, etc. are important to properly trace the evolution of the yielded material (e.g. Tenorio-Tagle 1996; de Avillez \& Mac Low 2002).

Therefore, a unique evolutionary scenario to describe the path from the creation of the new nitrogen to its incorporation to the warm ionized medium seems unlikely. In the following we propose some evolutionary paths for our knots $\sharp 1-3$. These scenarios do not intend to be more than reasonable suggestions that compile the constraints derived from our results and which could be tested with detailed modeling.

Regarding our knots $\sharp 2$ and $\sharp 3$, we hypothesize here about the possibility that they constitute two snapshots of the same evolutionary path. According to Harris et al. (2004) the main clusters associated to them are relatively similar in terms of mass and youth, although those in knot $\sharp 3$ are slightly older ${ }^{7}$.

\footnotetext{
7 Knot $\sharp 2$ corresponds to clusters 4 and 8 in Harris et al. (2004), with stellar masses of $\sim 2.7$ and $1.3 \times 10^{4} M_{\odot}$ and ages of 1 and 5 Myr while knot $\sharp 3$ corresponds to their cluster 3 and 5 , with masses 4.2 and $2.1 \times$ $10^{4} M_{\odot}$ and ages of 8 and $11 \mathrm{Myr}$, respectively.
}

Supporting this youth, candidates to supernova remnants have been found close to both knots (Labrie \& Pritchet 2006). Moreover, only knot $\sharp 2$ presented spectral features typical of Wolf-Rayet stars. In this evolutionary path, stars would expel their processed nitrogen at very early stages ( $\$ 5 \mathrm{Myr})$. The difference between the age of the cluster in knot $\sharp 3(\sim 10 \mathrm{Myr})$ and the moment when the yielding of extra-N took place (as soon as $\sim 2.5 \mathrm{Myr}$, according to the models of Mollá \& Terlevich 2012) sets an upper limit of $\sim 8$ Myr for the duration of this process. If rotating $\mathrm{O}$ stars were playing a relevant role, this limit could be extended up to the age of clusters. A lower limit can be estimated under the most optimistic (and efficient) assumption: instantaneous incorporation of the yielded material to the warm ionized gas. If, in addition, we assume that the velocity of the ionized gas with respect to knot $\sharp 3\left(\Delta v \sim 20 \mathrm{~km} \mathrm{~s}^{-1}\right.$, see Paper I) traces the velocity under which the contamination of extra nitrogen propagates through the ISM, the area could be enriched in only 2 Myr. Therefore, the process of the cooling down and mixing of the yielded material with the ISM over an area of $40-50 \mathrm{pc}$ in diameter should last between $\sim 2$ and $\sim 8$ Myr. Note that the moment when the newly created material has completely mixed with the previously existing gas, and any chemical inhomogeneity has been erased, should occur much later and cannot be delimited with these observations further than $\sim 10 \mathrm{Myr}$.

Knot $\sharp 1$ should follow a different evolutionary path since at this location both WR features and nitrogen enrichment are found. The knot is associated to two very young and massive super star clusters (e.g. Schaerer et al. 1999; Gorjian et al. 2001; Alonso-Herrero et al. 2004), embedded in a very dense and compact nebula (Turner et al. 2000). In that sense, they can be seen as nascent clusters that have not managed yet to disperse the cloud of gas where they were born. This implies a very particular set of physical conditions for the warm ISM. Indeed, a giant molecular cloud associated to this region has been reported (Meier et al. 2002) and we showed through this work that $n_{\mathrm{e}}$ and $T_{\mathrm{e}}$ associated to this region are relatively high, as well as the degree of ionization. Also, the region presents supersonic velocity widths and high extinction (Paper I). Somehow, the combination of some of these particular conditions has made possible the incorporation of the newly created nitrogen at an earlier stage.

A third example mentioned in previous work provides a different evolutionary path. García-Benito \& Pérez-Montero (2012) gives an age of $3.5 \mathrm{Myr}$ and a stellar mass of $\sim 5 \times 10^{3} M_{\odot}$ for their knot $\mathrm{C}$ in NGC 6789. Moreover, they report a relative abundance of nitrogen $\sim 0.2$ dex larger than in their other apertures. No WR feature was detected for this knot (García-Benito, priv. comm.). Clearly, at this mass regime, the yield is dominated by stochastic effects. Given the lack of any WR detection one has to resort to rotating $\mathrm{O}$ stars, or other massive star evolutionary stages, as the cause of the N-enhancement. Contrary to what happened with our knot $\sharp 1$ no particularly extreme physical conditions other than a complex inner dust structure were reported for its surrounding ISM. This leaves the open question of how this ISM managed to incorporate this new material in such a short time scale.

All in all, even if there seems to be a connection between WR emission and nitrogen enhancement, as supported by the fact that WR galaxies show an elevated N/O relative to nonWR galaxies (Brinchmann et al. 2008), local examples like NGC 5253 and NGC 6789, where linear spatial resolutions of $\sim 20 \mathrm{pc} \operatorname{arcsec}^{-1}$ can be achieved, show how this relationship is complex. Specifically, the case of the knots $\sharp 2$ and $\sharp 3$ in NGC 5253, where WR emission and N-enhancement are associated to different star clusters separated by only $\sim 90 \mathrm{pc}$, illustrates 
the possibility that in galaxies at distances $\gtrsim 25 \mathrm{Mpc}$, the spatial coincidence of WR emission and N/O overabundances does not necessarily imply an intimate association between them based on cause-effect relationship. However, this does not reject the possibility that both of them were related due to a common external cause. As an example, in NGC 5253 one could identify as this external cause the putative event that triggered the starburst (i.e. the past interaction with M 83).

\section{Conclusions}

We have carried out a detailed 2D study of the physical and chemical properties of the ionized gas in the central part of NGC 5253, a very nearby BCD. The area was mapped in a continuous manner with the ARGUS-IFU unit of FLAMES. This work represents the natural continuation of the one presented in Paper I. The different maps utilized along the paper as well as the reduced data cubes are available as FITS files from the authors.

The major conclusions can be summarized as follows:

1. Physical and chemical properties of the ionized gas associated to the main star clusters were derived by extracting spectra in apertures of 9-10 spaxels. Measurements associated to knots $\sharp 1$ and $\sharp 2$ agree in general with those previously reported. The existing discrepancies are associated to the assumed electron temperature. Abundances for knot $\sharp 3$ (not reported so far) are also provided. With the exception of the relative abundance for $\mathrm{N} / \mathrm{O}$ in knot $\sharp 1$ and possibly knot $\sharp 3$, no chemical species seems overabundant in any of the selected apertures.

2. Maps of the electron density based on four different tracers - namely [O II], [S II], [Fe III], and [Ar IV] line ratios - were discussed. In all the cases, higher densities are associated to the main GH nR. The joint analysis of these maps is consistent with a 3D stratified view of the nebula where the highest densities are located in the innermost layers while density decreases when going outwards.

3. The $2 \mathrm{D} n_{\mathrm{e}}$ structure for the two main kinematic components was also derived using the [O II] lines as baseline. While the so-called broad component follows the picture described above, there is a change of structure for the narrow one. This fits well with the proposed scenario in Paper I where this component was associated to a shell of previously existing material that had been piled up by the outflow associated to the broad component.

4. Maps for $T_{\mathrm{e}}([\mathrm{S} \mathrm{II}])$ and $T_{\mathrm{e}}([\mathrm{O} \mathrm{III}])$ were derived. To our knowledge, this is the first time that a $T_{\mathrm{e}}\left(\left[\mathrm{S}_{\mathrm{II}}\right]\right)$ map for an extragalactic object is presented. Also, we have provided one of the few examples of $T_{\mathrm{e}}([\mathrm{O} \mathrm{III}])$ maps to date. The joint interpretation of the information on the plane of the sky and along the line of sight is consistent with a $T_{\mathrm{e}}$ structure in $3 \mathrm{D}$ with higher temperatures close to the main ionizing source surrounded by a colder and more diffuse component. This $T_{\mathrm{e}}$ structure together with the lack of any strong broad component far from the main GH IIR (Paper I) is in accord with the lack of any clear evidence of shocks playing a dominant role.

5. Ionization structure was traced by means of the $[\mathrm{O}$ III $] \lambda \lambda 4959, \quad 5007 /[\mathrm{O}$ II $] \lambda \lambda 3726, \quad 3729, \quad[\mathrm{O}$ II $] \lambda \lambda 3726$, $3729 / \mathrm{H} \beta$, and $[\mathrm{S} \mathrm{II}] \lambda \lambda 6717,6731 / \mathrm{H} \alpha$ ratios. The first two predict similar ionization degree, while the third one would be typical of lower ionization. A possible 3D interpretation of both the observed structure in the maps for each individual ratio and the discrepancy of $\left[\mathrm{S}_{\mathrm{II}}\right] \lambda \lambda 6717,6731 / \mathrm{H} \alpha$ for individual spaxels is consistent with the lower ionization species (i.e. $\mathrm{S}^{+}$) delineating the more extended diffuse component.

6. Maps for the 2D distribution of abundances for oxygen, neon, argon were derived. All of them are consistent with no chemical inhomogeneities. The derived mean $( \pm$ standard deviation) oxygen abundance is $12+\log (\mathrm{O} / \mathrm{H})=8.26 \pm 0.04$. The mean logarithmic relative abundances for argon and neon were $-0.65 \pm 0.03$ and $-2.33 \pm 0.06$, respectively.

7. In the same manner, a map for the $2 \mathrm{D}$ distribution of nitrogen was derived. NGC 5253 typically presents a $\log (\mathrm{N} / \mathrm{O})$ of $\sim-1.32 \pm 0.05$. However, there are two locations with enhanced N/O. The first one was already reported and characterized. With a $\log (\mathrm{N} / \mathrm{O}) \sim-0.95$, it occupies an elliptical area of about $80 \mathrm{pc} \times 35 \mathrm{pc}$ and is associated to the two SSCs at the nucleus of the galaxy. The second one is reported here for the first time. It presents a $\log (\mathrm{N} / \mathrm{O}) \sim-1.17$ and it is associated to two moderately massive $\left(2-4 \times 10^{4} M_{\odot}\right)$ and relatively old $(\sim 10 \mathrm{Myr})$ clusters (knot $\sharp 3$ ).

8. The map of N/O relative abundance derived through the direct method was compared with those derived using strong line methods. The comparison supports a method based on $\mathrm{N} 2 \mathrm{O} 2$ over a method based on N2S2 in the search of chemical inhomogeneities within a galaxy since the first method is sensitive to a wider range of N/O abundances and is more stable against variations of physical conditions within the area of interest.

9. We utilized the results on the localized detection of WR emission and nitrogen enhancement to compile and discuss the factors that affect the complex relationship between the presence of WR stars and N/O excess. Even if such a relationship is apparent, a unique scenario describing the path from the production of the new nitrogen to its incorporation into the warm ionized medium seems unlikely. In particular, we use the areas associated to knots $\sharp 2$ and $\sharp 3$ in NGC 5253 as examples of WR emission and $\mathrm{N}$-enhancement that would be perceived as spatially coincident at distances $\gtrsim 25 \mathrm{Mpc}$, but that are not intimately associated in a cause-effect fashion. However, this does not refute the possibility that both of them are related due to a common external cause.

Acknowledgements. This paper has benefited from fruitful conversations during the Workshop "Metals in 3D: New insights from Integral Field Spectroscopy". We would like to thank in particular to R. García-Benito, B. James, M. Mollá, L. Smith, G. Tenorio-Tagle, and E. Pérez-Montero and M. Relaño as well as to M. Westmoquette and R. Amorín with whom we shared stimulating discussions that helped to improve it. We also thank the referee for the useful comments that have significantly improved the first submitted version of this paper. Based on observations carried out at the European Southern Observatory, Paranal (Chile), programmes 078.B-0043(A) and 383.B-0043(A). This paper uses the plotting package jmaplot, developed by Jesús Maíz-Apellániz, http://dae45.iaa.csic.es: 8080/ jmaiz/software. This research made use of the NASA/IPAC Extragalactic Database (NED), which is operated by the Jet Propulsion Laboratory, California Institute of Technology, under contract with the National Aeronautics and Space Administration. The STARLIGHT project is supported by the Brazilian agencies CNPq, CAPES, FAPESP and by the France-Brazil CAPES-COFECUB programme. A.M.-I. is supported by the Spanish Research Council within the programme JAE-Doc, Junta para la Ampliación de Estudios, co-funded by the FSE. This work has been partially funded by the projects AYA2010-21887 from the Spanish PNAYA, CSD2006 00070 "1st Science with GTC" from the CONSOLIDER 2010 programme of the Spanish MICINN, and TIC114 Galaxias y Cosmología of the Junta de Andalucía (Spain).

\section{References}

Alloin, D., Collin-Souffrin, S., Joly, M., \& Vigroux, L. 1979, A\&A, 78, 200 Alonso-Herrero, A., Takagi, T., Baker, A. J., et al. 2004, ApJ, 612, 222 
Amorín, R., Pérez-Montero, E., Vílchez, J. M., \& Papaderos, P. 2012, ApJ, 749, 185

Asplund, M., Grevesse, N., Sauval, A. J., Allende Prieto, C., \& Kiselman, D. 2004, A\&A, 417, 751

Brinchmann, J., Kunth, D., \& Durret, F. 2008, A\&A, 485, 657

Bruzual, G., \& Charlot, S. 2003, MNRAS, 344, 1000

Calzetti, D., Harris, J., Gallagher, III, J. S., et al. 2004, AJ, 127, 1405

Campbell, A., Terlevich, R., \& Melnick, J. 1986, MNRAS, 223, 811

Cardelli, J. A., Clayton, G. C., \& Mathis, J. S. 1989, ApJ, 345, 245

Cid Fernandes, R., Mateus, A., Sodré, L., Stasińska, G., \& Gomes, J. M. 2005, MNRAS, 358, 363

Cid Fernandes, R., Schoenell, W., Gomes, J. M., et al. 2009, Rev. Mex. Astron. Astrofis. Conf. Ser., 35, 127

Crowther, P. A. 2007, ARA\&A, 45, 177

de Avillez, M. A., \& Mac Low, M.-M. 2002, ApJ, 581, 1047

De Robertis, M. M., Dufour, R. J., \& Hunt, R. W. 1987, JRASC, 81, 195

Denicoló, G., Terlevich, R., \& Terlevich, E. 2002, MNRAS, 330, 69

Díaz, A. I., Castellanos, M., Terlevich, E., \& Luisa García-Vargas, M. 2000, MNRAS, 318, 462

Ercolano, B., Dale, J. E., Gritschneder, M., \& Westmoquette, M. 2012, MNRAS, 420,141

Fernández-Martín, A., Martín-Gordón, D., Vílchez, J. M., et al. 2012, A\&A, 541, A119

García-Benito, R. 2009, Ph.D. Thesis, Universidad Autónoma de Madrid, Spain

García-Benito, R., \& Pérez-Montero, E. 2012, MNRAS, 423, 406

García-Lorenzo, B., Cairós, L. M., Caon, N., Monreal-Ibero, A., \& Kehrig, C. 2008, ApJ, 677, 201

Garnett, D. R. 1990, ApJ, 363, 142

Garnett, D. R. 1992, AJ, 103, 1330

Girardi, L., Bressan, A., Bertelli, G., \& Chiosi, C. 2000, A\&AS, 141, 371

Gorjian, V., Turner, J. L., \& Beck, S. C. 2001, ApJ, 554, L29

Grevesse, N., \& Anders, E. 1989, in Cosmic Abundances of Matter, ed. C. J. Waddington, AIP Conf. Ser., 183, 1

Guseva, N. G., Izotov, Y. I., \& Thuan, T. X. 2000, ApJ, 531, 776

Guseva, N. G., Izotov, Y. I., Stasińska, G., et al. 2011, A\&A, 529, A149

Hägele, G. F., Pérez-Montero, E., Díaz, Á. I., Terlevich, E., \& Terlevich, R. 2006, MNRAS, 372, 293

Harris, J., Calzetti, D., Gallagher, III, J. S., Smith, D. A., \& Conselice, C. J. 2004, ApJ, 603, 503

Izotov, Y. I., Thuan, T. X., \& Lipovetsky, V. A. 1994, ApJ, 435, 647

Izotov, Y. I., Schaerer, D., Blecha, A., et al. 2006a, A\&A, 459, 71

Izotov, Y. I., Stasińska, G., Meynet, G., Guseva, N. G., \& Thuan, T. X. 2006b, A\&A, 448, 955

James, B. L., Tsamis, Y. G., Barlow, M. J., et al. 2009, MNRAS, 398, 2

Karachentsev, I. D., Tully, R. B., Dolphin, A., et al. 2007, AJ, 133, 504

Keenan, F. P., Aller, L. H., Ryans, R. S. I., \& Hyung, S. 2001, PNAS, 98, 9476

Kehrig, C., Vílchez, J. M., Sánchez, S. F., et al. 2008, A\&A, 477, 813

Kewley, L. J., \& Dopita, M. A. 2002, ApJS, 142, 35

Kewley, L. J., \& Ellison, S. L. 2008, ApJ, 681, 1183

Kingsburgh, R. L., \& Barlow, M. J. 1994, MNRAS, 271, 257

Kobulnicky, H. A., \& Skillman, E. D. 2008, AJ, 135, 527

Kobulnicky, H. A., Skillman, E. D., Roy, J.-R., Walsh, J. R., \& Rosa, M. R. 1997, ApJ, 477, 679

Kobulnicky, H. A., Kennicutt, Jr., R. C., \& Pizagno, J. L. 1999, ApJ, 514, 544

Kunth, D., \& Östlin, G. 2000, A\&A Rev., 10, 1

Labrie, K., \& Pritchet, C. J. 2006, ApJS, 166, 188

López-Sánchez, Á. R., \& Esteban, C. 2010, A\&A, 517, A85
López-Sánchez, Á. R., Esteban, C., García-Rojas, J., Peimbert, M., \& Rodríguez, M. 2007, ApJ, 656, 168

López-Sánchez, Á. R., Koribalski, B. S., van Eymeren, J., et al. 2012, MNRAS, 419, 1051

Maeder, A., \& Meynet, G. 2000, ARA\&A, 38, 143

Maeder, A., \& Meynet, G. 2010, New A Rev., 54, 32

Markwardt, C. B. 2009, in ASP Conf. Ser. 411, eds. D. A. Bohlender, D. Durand, \& P. Dowler, 251

Meier, D. S., Turner, J. L., \& Beck, S. C. 2002, AJ, 124, 877

Meynet, G., \& Maeder, A. 2005, A\&A, 429, 581

Mollá, M., \& Terlevich, R. 2012, MNRAS, accepted [arXiv: 1204 .4879]

Mollá, M., Vílchez, J. M., Gavilán, M., \& Díaz, A. I. 2006, MNRAS, 372, 1069

Monreal-Ibero, A., Arribas, S., \& Colina, L. 2006, ApJ, 637, 138

Monreal-Ibero, A., Arribas, S., Colina, L., et al. 2010a, A\&A, 517, A28

Monreal-Ibero, A., Vílchez, J. M., Walsh, J. R., \& Muñoz-Tuñón, C. 2010b, A\&A, 517, A27 (Paper I)

Monreal-Ibero, A., Relaño, M., Kehrig, C., et al. 2011a, MNRAS, 413, 2242

Monreal-Ibero, A., Vílchez, J. M., Walsh, J., \& Muñoz-Tuñon, C. 2012, Dwarf Galaxies: Keys to Galaxy Formation and Evolution, Proc. Symp. 3 JENAM 2010 (Berlin Heidelberg: Springer-Verlag), eds. P. Papaderos, S. Recchi, \& G. Hensler, 215

Núñez-Díaz, M., Mesa-Delgado, A., Esteban, C., et al. 2012, MNRAS, 421, 3399

Osterbrock, D. E., \& Ferland, G. J. 2006, Astrophysics of gaseous nebulae and active galactic nuclei, eds. D. E. Osterbrock, \& G. J. Ferland

Pagel, B. E. J., Edmunds, M. G., Blackwell, D. E., Chun, M. S., \& Smith, G. 1979, MNRAS, 189, 95

Pagel, B. E. J., Simonson, E. A., Terlevich, R. J., \& Edmunds, M. G. 1992, MNRAS, 255, 325

Pasquini, L., Ávila, G., Blecha, A., et al. 2002, The Messenger, 110, 1

Pérez-Montero, E., \& Contini, T. 2009, MNRAS, 398, 949

Pérez-Montero, E., \& Díaz, A. I. 2003, MNRAS, 346, 105

Pérez-Montero, E., García-Benito, R., Hägele, G. F., \& Díaz, Á. I. 2010, MNRAS, 404, 2037

Pérez-Montero, E., Relaño, M., Vílchez, J. M., \& Monreal-Ibero, A. 2011, MNRAS, 412, 675

Pradhan, A. K. 1976, MNRAS, 177, 31

Pustilnik, S., Kniazev, A., Pramskij, A., et al. 2004, A\&A, 419, 469

Relaño, M., Monreal-Ibero, A., Vílchez, J. M., \& Kennicutt, R. C. 2010, MNRAS, 402, 1635

Sabbadin, F., Minello, S., \& Bianchini, A. 1977, A\&A, 60, 147

Schaerer, D., Contini, T., \& Pindao, M. 1999, A\&AS, 136, 35

Shaw, R. A., \& Dufour, R. J. 1995, PASP, 107, 896

Sidoli, F. 2010, Ph.D. Thesis, University of London, UK

Stasińska, G. 1990, A\&AS, 83, 501

Storey, P. J., \& Hummer, D. G. 1995, MNRAS, 272, 41

Tenorio-Tagle, G. 1996, AJ, 111, 1641

Thuan, T. X., Izotov, Y. I., \& Lipovetsky, V. A. 1996, ApJ, 463, 120

Tsamis, Y. G., Walsh, J. R., Péquignot, D., et al. 2008, MNRAS, 386, 22

Turner, J. L., Beck, S. C., \& Ho, P. T. P. 2000, ApJ, 532, L109

van den Bergh, S. 1980, PASP, 92, 122

Villaverde, M., Cerviño, M., \& Luridiana, V. 2010, A\&A, 517, A93

Walsh, J. R., \& Roy, J.-R. 1987, ApJ, 319, L57

Walsh, J. R., \& Roy, J.-R. 1989, MNRAS, 239, 297

Welch, G. A. 1970, ApJ, 161, 821

Westmoquette, M. S., Gallagher, J. S., \& de Poitiers, L. 2010, MNRAS, 403, 1719

Zeippen, C. J. 1982, MNRAS, 198, 111 